\title{
Stichwort: Digitalisierung in der kulturell-ästhetischen Bildung - eine konfigurierende Forschungssynthese
}

\author{
Stephan Kröner (D) Alexander Christ • Marcus Penthin
}

Eingegangen: 4. März 2020 / Überarbeitet: 3. August 2020 / Angenommen: 11. Januar 2021 / Online publiziert: 16. Februar 2021

(C) Der/die Autor(en) 2021

Zusammenfassung Digitalisierung verändert zunehmend die kulturelle Bildung. Im Zuge des technologischen Fortschritts werden auch soziale Interaktionen, ästhetische Erfahrungen und Ausdrucksformen transformiert. In diesem Beitrag wird über eine konfigurierende Forschungssynthese zur Kartierung des sich entwickelnden Forschungsfeldes zur Digitalisierung in der kulturellen Bildung berichtet. Dazu wurden im Fachportal Pädagogik und in Scopus empirisch ausgerichtete Originalarbeiten mit explizitem Bezug zu Digitalisierung in der kulturellen Bildung identifiziert und nach formalen sowie inhaltlichen Kriterien kategorisiert. Inhaltlich wird ein Schwerpunkt in den bildenden Künsten deutlich. Arbeiten zu aktuellen Entwicklungen wie Digital Making und Virtual Reality sind jedoch selten. Ein Großteil der vorliegenden Arbeiten fokussiert auf formale Bildungsprozesse. Im Zentrum steht oft die Frage nach Determinanten von Kompetenzerwerb, Interesse und Motivation. Resultierende Evidenz wird jedoch häufig begrenzt durch schwache Designs, naive Vergleiche analog/digital und unterausgewertete Datensätze. Diskutiert wird, wie diese Desiderate in künftiger quantitativer wie qualitativer Forschung adressiert werden können.

Schlüsselwörter Digitalisierung · Forschungssynthese · Kulturelle Bildung · Ästhetische Bildung · Kulturelle Aktivitäten

Prof. Dr. S. Kröner $(\bowtie) \cdot$ A. Christ, M.A. · M. Penthin Lehrstuhl für Empirische Bildungsforschung, FAU Erlangen-Nürnberg, Regensburger Str. 160, 90478 Nürnberg, Deutschland E-Mail: stephan.kroener@fau.de

A. Christ, M.A.

E-Mail: alexander.chrale.christ@fau.de

M. Penthin

E-Mail: marcus.penthin@fau.de 


\title{
Digitalization in aesthetics, arts and cultural education-a scoping review
}

\begin{abstract}
Digitalization is increasingly changing arts and cultural education. Along with this technological progress comes a transformation of social interactions, aesthetic experiences and forms of expression. This paper presents a scoping review for mapping the evolving research field of digitalization in aesthetic, arts and cultural education (D-ACE). For this, the databases Fachportal Pädagogik and Scopus were exploited to identify original empirical research papers with explicit reference to D-ACE, which were then categorized according to formal and content-related criteria. This showed an emphasis on the visual arts; contrasting with a lack of research on current developments in fields such as digital making and virtual reality. Most of the relevant studies concentrate on formal educational processes. The overall focus on determinants of competence acquisition, interest and motivation was frequently limited by weak study designs, simplistic comparisons between analog and digital, and insufficiently analyzed data sets. We discuss how these desiderata can be addressed with quantitative and qualitative research in the future.
\end{abstract}

Keywords Aesthetic education · Arts education · Digitalization · Research synthesis $\cdot$ Review

\section{Einleitung}

Die Zahl empirischer Publikationen im weiten Feld der Bildungsforschung steigt seit längerem von Jahr zu Jahr (Hasselhorn und Krampen 2019). Es ergeben sich beständig aktuelle bildungsrelevante technische Entwicklungen und gesellschaftliche Herausforderungen, die empirische Forschung anregen. Um einen Überblick über den stetig wachsenden Korpus von Arbeiten im sich zunehmend ausdifferenzierenden Feld der Empirischen Bildungsforschung zu ermöglichen, sind Forschungssynthesen hilfreich. Sie leisten eine Kartierung der Arbeiten nach formalen und inhaltlichen Kriterien, bereiten die dazu vorliegende Evidenz auf und identifizieren Desiderate für die weitere Forschung. Ein sich zunehmend etablierender Teilbereich der Empirischen Bildungsforschung ist das Feld der Digitalisierung in der kulturellen Bildung: Es liegt am Schnittpunkt von Herausforderungen und Potenzialen, die sich aus zunehmender Unterschiedlichkeit von Individuen und gesellschaftlichen Gruppen ebenso ergeben wie aus rasant voranschreitender technologischer Entwicklung (Kröner et al. 2019). Für diesen Bereich zielt das Forschungsvorhaben DiKuBiMeta darauf ab, quantitativ-empirische Forschung zu aktuellen Forschungsschwerpunkten zu identifizieren, zu kartieren und zu synthetisieren. Demgemäß wird in der vorliegenden Arbeit eine - auch qualitative Arbeiten einschließende - konfigurierende Forschungssynthese von empirischen Arbeiten dokumentiert, die sich explizit auf die kulturelle Bildung beziehen und Phänomene der Digitalisierung thematisieren. Die identifizierten Arbeiten werden nach formalen wie inhaltlichen Kriterien kategorisiert und es wird eine Einschätzung der Qualität dazu jeweils vorliegender Evidenz ermöglicht. In der Folge legen wir zunächst unser Verständnis von Digita- 
lisierung, Kultur und Bildung dar. Anschließend diskutieren wir die Bedeutung von empirischer Forschung zur Digitalisierung in der kulturellen Bildung. Nach einer kurzen Einführung in Forschungssynthesen im Allgemeinen sowie konfigurierende Forschungssynthesen im Besonderen geben wir schließlich einen Überblick über die von uns bearbeiteten Forschungsfragen und berichten im empirischen Teil über die abgedeckten empirischen Arbeiten an der Schnittstelle von Digitalisierung und kultureller Bildung.

\section{Digital - Kultur - Bildung - Begriffsbestimmungen}

Digitale Technik, Digitalisierung: Unter Technik im Allgemeinen verstehen wir im Folgenden künstliche, gegenständliche Sachsysteme einschließlich der auf ihre Entstehung und Verwendung bezogenen menschlichen Handlungen (VDI 2000). Der Begriff Digitale Technik im Besonderen bezieht sich auf die softwaregesteuerte Verarbeitung computerlesbarer Daten unter Einbezug von Computernetzwerken bzw. einzelnen Computern mit Interfaces zur Ein- und Ausgabe von Daten (vgl. Jörissen und Unterberg 2019). Jenseits der rein technischen Aspekte ist für die Digitalisierung in der kulturellen Bildung vor allem bedeutsam, dass digitale Technik im Rahmen sprunghafter, non-linearer Entwicklungen mittlerweile alle Bereiche von Gesellschaft und täglichem Leben erreicht hat und diese zunehmend durchdringt (BMBF 2017; Couldry 2014; Grenz und Pfadenhauer 2017). Derartige Entwicklungen verändern nicht nur Arbeitswelt und Kommunikationsprozesse (Krotz 2017), sondern auch informelle Bildungsprozesse. Dies betrifft Freizeitbeschäftigungen wie das Hören von Musik (Wehner et al. 2017) oder das Spielen von Videospielen (Abend und Beil 2017) ebenso wie formale und non-formale Bildungsprozesse (Breiter et al. 2012). Diese eher sozialen Implikationen der Digitalisierung jenseits rein technischer Aspekte werden im Mediatisierungsdiskurs thematisiert (Hugger 2013; Krotz 2017; Krotz et al. 2017; Krotz und Hepp 2012). Mediatisierungsprozesse haben auch kulturelle Angebote und deren Nutzung grundlegend verändert (Hepp und LehmannWermser 2013). Vor allem aber schaffen sie die technischen Voraussetzungen für neue Ausdrucksformen, soziale Handlungsmuster und globale Interaktionen (Jörissen et al. 2019). Für eine grobe Differenzierung von Effekten der Digitalisierung können die zugrunde liegenden technologischen Entwicklungen nach Zeitpunkt ihres Populärwerdens unterschieden werden. Diese bezeichnen wir im Folgenden als Phänomene der ersten, zweiten und dritten Digitalisierungsgeneration. Die erste Digitalisierungsgeneration umfasst Stand-Alone-Software sowie Web 1.0 „Read-Only“Anwendungen. Die zweite Digitalisierungsgeneration bezieht sich auf soziale Netzwerke und andere Web 2.0-Anwendungen mit nutzergenerierten Inhalten. Die dritte Digitalisierungsgeneration schließlich bezieht sich auf Phänomene wie Digital Making sowie Virtual und Augmented Reality und auf weitere aktuelle Entwicklungen im Kontext von Web 3.0 (z.B. „Executable-Web“-Anwendungen). Die gewählten Bezeichnungen für die Digitalisierungsgenerationen berücksichtigen, dass unsere Kategorien mit den häufig thematisierten Unterscheidungen zwischen Web-Generationen (Web X.0, Benito-Osorio et al. 2013) zwar überlappen, aber nicht identisch sind, da insbesondere bei den von uns den Generationen 1 und 3 zugeordneten Phä- 
nomenen eine Internetanbindung nicht zwingend erforderlich ist (vgl. Benito-Osorio et al. 2013; Choudhury 2014 und Tromp et al. $2018 \mathrm{zu}$ den, jenseits von Web 3.0 heterogenen, Einteilungen in Web-Generationen). Unabhängig von der Zuordnung zu Digitalisierungsgenerationen und unabhängig von der jeweiligen Gewichtung von Hard- und Software verwenden wir im Folgenden durchgängig den Begriff des digitalen Werkzeugs (Frese und Brodbeck 1989), da diese Metapher der Rolle gerecht wird, die Hard- und Software bei der Rezeption, Produktion und Kreation kultureller Artefakte spielt.

Bildung: Unsere Definition von Bildung legen wir im Folgenden einer breiten Recherche von Studien zur Digitalisierung in der kulturellen Bildung zugrunde. Deshalb umfasst die Definition selbstbestimmtes Handeln mit dem Potenzial zur adaptiven Veränderung der Person ebenso wie Angebote zur Förderung solchen Handelns und die aus den Prozessen resultierenden Veränderungen auf Seiten der Person. Damit schließt sie in dreierlei Hinsicht an verbreitete Definitionen an. Diese fassen (1) Bildung als Prozess, in dessen Verlauf eine Person im Idealfall darauf hinarbeitet, alles, was ,die Welt um ihn her und sein inneres Selbst darbietet, mit allen Werkzeugen seiner Empfänglichkeit in sich aufzunehmen und mit allen Kräften seiner Selbsttätigkeit umzugestalten und sich anzueignen“ (Humboldt [1799] 1904, S. 117; ohne Beschränkung der Allgemeinheit hinsichtlich des Geschlechts). Bildung in diesem Sinne meint also, sich ins Verhältnis zu setzen zu sich selbst in der Zeit, zu anderen in der Gemeinschaft und zu den Sachen und Sachverhalten in der Welt (Iske und Meder 2010). Außerdem ist für Bildungsdefinitionen (2) die Angebotsseite relevant, da Bildungsprozesse nicht nur spontan ablaufen, sondern auch durch gezielt gestaltete Situationen gefördert werden können, die darauf ausgerichtet sind, den Handelnden zu einer geeigneten „Ausstattung zum Verhalten in der Welt“ zu verhelfen (Robinsohn 1971, S. 231, zit. n. Kuypers und Leyendecker 1982, S. 72). Schließlich stellt Bildung (3) als Ergebnis ,die subjektive Seite der Kultur dar ... in lebendige Anschauungen und Kräfte umgesetzt" (Krieck 1930, S. 79). Diese Definition von Bildung ist anschlussfähig an den Diskurs um Medien und Bildung im post-digitalen Zeitalter, d. h. ,,unter Bedingungen vielfältiger und komplexer digitaler Transformationen und Disruptionen“ (Jörissen 2018, S. 51). Zwar resultieren aus der Mediatisierung des Alltags mannigfaltige Veränderungen in kulturellen und sozialen Interaktions-, Artikulations- und Partizipationsformen; dennoch geht es auch bei digitaler Bildung nach wie vor um das - allerdings post-digital transformierte Verhältnis zu Selbst, Gemeinschaft und Welt (Hugger 2013; Iske und Meder 2010). Ebenso dürften für digitale Bildung, nicht anders als für analoge Bildung, Effekte auf die grundlegenden Persönlichkeitseigenschaften, wie sie in der Psychologie als Big Five operationalisiert werden (Costa und McCrae 1995), schwächer sein als allgemein angenommen. Gleichwohl kann sie handlungswirksam werden, indem sie sich in Änderungen charakteristischer Anpassungen oder des narrativen Selbst niederschlägt und schließlich in verändertem Verhalten gegenüber der Umwelt resultiert (vgl. Kröner et al. 2008; McAdams 2006). Bildung in diesem Sinne zeigt sich, wenn es Menschen gelingt, ihr „Ich mit der Natur in die allgemeinste, regste und übereinstimmendste Wechselwirkung zu bringen“" (Humboldt [1799] 1904, S. 117).

Kultur: Es existiert eine große Zahl von Definitionen sowohl zum Kulturbegriff als auch für die kulturelle Bildung (Bamford 2010, S. 68 ff.; Ijdens 2016; Reckwitz 
2004). Nach wie vor häufig zitiert wird die klassische Definition Tylors von Kultur als ,....] that complex whole which includes knowledge, belief, art, morals, law, customs, and many other capabilities and habits acquired by man as a member of society“ (Tylor [1871] 2010, S. 1). Abweichend von dieser breiten Definition ist für die Community der kulturellen Bildungsforschung eher ein enges Kulturverständnis prototypisch, das auf Kultur im Sinne von Künsten fokussiert (vgl. Projektgruppe „Forschung zur Kulturellen Bildung in Deutschland“ 2014). Zugleich wird darin häufig Bezug auf pädagogische Praxisfelder genommen, wie sie zum Beispiel im Dachverband Bundesvereinigung kulturelle Kinder- und Jugendbildung (BKJ) abgebildet sind. Da in der vorliegenden Arbeit kulturelle Bildung im Sinne des Selbstverständnisses der entsprechenden Forschungscommunity im Zentrum steht, lehnen wir uns an dieses Kulturverständnis an. Dabei orientieren wir uns an der klassischen Unterteilung der Künste in Literatur, Musik, bildende und darstellende Kunst (vgl. Kristeller 1951, zu dieser ähnlich bereits bei Batteux [1747] 1976, aufzufindenden Einteilung). Um jedoch nicht von vornherein einen Großteil potenziell relevanter Arbeiten mit Bezug zur Digitalisierung auszuschließen, fassen wir diese so weit auf, dass Digitalisierungsphänomene wie künstlerisch konnotierte Interaktionen in sozialen Netzwerken davon jeweils ebenso eingeschlossen sind wie kreative Aktivitäten im Kontext virtueller Realitäten.

Empirische Forschung zur Digitalisierung in der Kulturellen Bildung: Alle im Folgenden erörterte Begriffe ergeben sich ohne weiteres aus der Kombination der bereits definierten Bestandteile. Als spezifische Form von Bildung umfasst kulturelle Bildung (1) Bildungsprozesse ,im Medium der Künste (Liebau et al. 2013; Rat für kulturelle Bildung 2013, S. 15), (2) Bildungsangebote, welche auf die Anregung von ästhetischen Erfahrungen oder von Lernprozessen in den Künsten abzielen (Fink et al. 2012, S. 12) sowie (3) eine daraus resultierende veränderte „Ausstattung zum Verhalten“ in den Künsten (Robinsohn 1971, S. 231, zit. n. Kuypers und Leyendecker 1982, S. 72; mit Einschränkung gilt diese Definition auch im englischem Sprachraum, wobei sich ,cultural education“ eher auf kulturelles Lernen bezieht). Kulturelle Bildung schlägt sich schließlich in veränderten Aktivitäten im Feld der Künste nieder (vgl. Kröner 2013). Zur digitalen kulturellen Bildung wird kulturelle Bildung dann, wenn kulturelle Bildungsprozesse oder Bildungsangebote die Interaktion mit digital transformierten kulturellen Umwelten beinhalten oder die Ergebnisse das Potenzial für das Verhalten zur Interaktion mit derartigen Umwelten verändern. Empirische Forschung zur Digitalisierung in der kulturellen Bildung umfasst alle publizierten und über einschlägige Datenbanken auffindbaren Forschungsarbeiten zur Digitalisierung in der kulturellen Bildung, die empirische Methoden verwenden. Diese Arbeiten können Bezüge zu qualitativen und quantitativen oder gemischten Analysen aufweisen. Quantitative Studien verwenden dabei typischerweise inferenzstatistische Analyseverfahren. Die analysierten quantitativen Daten wiederum können über Studien mit unterschiedlichen Designs gewonnen worden sein. Dabei kann es sich erstens um beobachtende, auf die Generierung von Beschreibungswissen abzielende Designs innerhalb eines einzelnen Messzeitpunkts handeln. Dies schließt entwicklungspsychologische Querschnittsstudien über mehrere Altersgruppen hinweg ebenso ein wie Studien, die Korrelationen lediglich innerhalb einer Altersgruppe untersuchen. Beide werden im Folgenden vereinfachend als querschnittlich 
bezeichnet. Zweitens können die Daten mittels beobachtender Designs über mehrere Messzeitpunkte hinweg gewonnen worden sein und die Generierung von Vorhersagewissen ermöglichen. Sie werden in Folgenden als längsschnittlich bezeichnet. Und drittens können sie mit Hilfe quasiexperimenteller oder experimenteller Designs gewonnen worden sein. Sie werden im Folgenden als Interventionsdesigns bezeichnet, wobei wir einen weitgefassten Interventionsbegriff verwenden, welcher die Treatments in eher grundlagenorientierten Experimenten ebenso einbezieht wie die Interventionen in quasiexperimentellen oder experimentellen Feldstudien. Diese Designs zielen auf die Gewinnung von Erklärungs- und Veränderungswissen ab (vgl. Bromme et al. 2014; Hussy et al. 2013; Thiese 2014).

Weitere Aspekte: Unsere Definition von Forschung zu kultureller Bildung ist breit gefasst. Sie schließt Aktivitäten mit Bezug zu den unterschiedlichsten Schichten oder Milieus ein (z. B. Hochkultur-, Spannungs- und Trivialschema, Schulze 2000) Weiterhin selektiert sie nicht nach Formalisierungsgrad, sondern umfasst formale, nonformale oder informelle Bildungsprozesse gleichermaßen (La Belle 1982). Auch in Bezug auf Lebensphasen nehmen wir keine Einschränkung vor (Hurrelmann 2003). Lediglich im Hinblick auf den Aktivitätsgrad richten wir den Fokus ausschließlich auf aktive und kreative Partizipation: Arbeiten zu rezeptiver kultureller Partizipation wie das reine Hören von Musik über Internet-Plattformen oder das Lesen in digitalem Format vorliegender Texte finden keine Berücksichtigung (Kröner 2013; in Anlehnung an Brickenkamp 1990). Wesentlich für die Einbeziehung einer Arbeit ist zudem, dass die Autoren selbst explizit auf kulturelle Bildung oder verwandte Begriffe wie ästhetische oder künstlerische Bildung Bezug nehmen und sich so dem Feld der kulturellen Bildung im engeren Sinne zuordnen.

\section{Empirische Forschung zur kulturellen Bildung vor dem Hintergrund der Digitalisierung}

Kulturelle Bildung im Allgemeinen im Sinne unserer breiten Definition wird seit geraumer Zeit empirisch erforscht. Dies zeigt sich in empirischen Zeitschriftenpublikationen zu Determinanten kultureller Aktivitäten (Hamari und Keronen 2017; Penthin et al. 2017; Schüller et al. 2017; Walmsley 2011), ebenso wie in solchen zu deren intendierten und beobachtbaren Effekten, beispielsweise im Kontext von Projekten wie ,Jedem Kind ein Instrument“ (Krupp-Schleußner und Lehmann-Wermser 2016) oder „Bildungsprozesse in der kulturellen Kinder- und Jugendarbeit“ (Stuckert et al. 2018). Zudem ist kulturelle Bildung Gegenstand aktueller BMBF-Förderschwerpunkte (BMBF 2019; Timm et al. 2020).

Auf kulturelle Bildung im formellen, schulischen Kontext beziehen sich insbesondere Arbeiten zu künstlerisch-musischen und sprachlichen Fächern. Beispiele dafür sind Studien zur Entwicklung von Selbstwirksamkeitserleben oder Kompetenzen in Bezug auf Aktivitäten, die auch für kulturelle Aktivitäten relevant sind (vgl. z. B. Troyer et al. 2019 oder Peura et al. 2019 für Literacy). Selbst wenn man Artikel ohne Bezug auf Digitalisierung einbezieht, findet sich dagegen deutlich weniger empirische Forschung zum non-formalen Kontext, obwohl gerade in der Freizeit ein maßgeblicher Teil der kulturellen Weltbegegnung von Kindern und Jugendli- 
chen angesiedelt ist und dort mit hohem intrinsischen Wert einhergeht (Kröner und Dickhäuser 2009; Valenzuela und Codina 2014).

Es liegen nur sehr wenige Forschungssynthesen im Feld der kulturellen Bildung vor (vgl. Rammstedt 2019 sowie Schröder 2019, basierend auf Daten von Sala und Gobet 2017, für erste Ansätze zu spezifischen Themen). Dies gilt verstärkt für die Schnittstelle von kultureller Bildung und Digitalisierung, die den Gegenstand der vorliegenden konfigurierenden Forschungssynthese darstellt. Orientierung bietende Synthesen erscheinen hier besonders gewinnbringend, da sich im Zuge der digitalen Transformation der Gesellschaft auch die von kultureller Bildungsforschung untersuchten Fragen nochmals neu und anders stellen: Sowohl die Umwelt als auch die Möglichkeiten zur Interaktion von Person und Umwelt verändern sich durch die Digitalisierung teilweise erheblich. Dies berührt auch Angebote, Prozesse und Ergebnisse im Rahmen von kultureller Bildung, weshalb diese aktuell einen interessanten Gegenstand empirischer Forschung darstellen (vgl. den BMBF-Förderschwerpunkt zur Digitalisierung in der Kulturellen Bildung, Jörissen et al. 2019). Empirische Forschung dazu, was kulturelle Bildung in einer digital transformierten Welt charakterisiert, umfasst sehr heterogene Phänomene. Diese Vielfalt ebenso wie die disziplinäre Fragmentierung der Forschung zur Digitalisierung in der kulturellen Bildung (z.B. Musikpädagogik, Informatik, Erziehungswissenschaft) spiegelt sich in einer Vielzahl theoretischer Bezüge und empirischer Zugänge wider. Diese gilt es zusammenzuführen, um das Potenzial von digitaler kultureller Bildung als Forschungsgebiet auszuschöpfen. Einen Schritt in diese Richtung möchten wir mit der vorliegenden konfigurierenden Forschungssynthese gehen. Sie soll die Arbeiten aus dem Feld der kulturellen Bildung gebündelt sichtbar machen und so auch deren Sichtbarkeit über die Grenzen des Feldes hinaus stärken.

\section{Forschungssynthesen und ihre Relevanz in der kulturellen Bildung}

Forschungssynthesen fassen Originalarbeiten in einem bestimmten Bereich oder zu einer spezifischen Fragestellung systematisch zusammen (Gough et al. 2017; Torgerson et al. 2017). Sie bilden die Grundlage sowohl für die Identifizierung von Forschungsdesideraten als auch für wissensbasiertes Handeln (Petticrew und Roberts 2012). Bei letzterem helfen sie, die Gefahr von Kurzschlüssen zu vermeiden, wie sie bei Entscheidungen auf der Basis eingeschränkter Informationen oder wegen Rückgriffen auf individuelle Erfahrungen oder Ansichten drohen (Dicks et al. 2014). Es lassen sich verschiedene Grundformen von Forschungssynthesen unterscheiden, die sich nach Gough et al. (2017) auf einem Kontinuum von aggregierend hin zu konfigurierend anordnen lassen. Während aggregierende Synthesen bzw. Metaanalysen meist auf homogene Arbeiten zu eng gesteckten Fragestellungen fokussieren und in der Berechnung von studienübergreifenden Kennwerten münden, ermöglichen konfigurierende Forschungssynthesen einen ordnenden Überblick über eine Vielzahl heterogener Studien nach bibliometrischen, methodischen und inhaltlichen Gesichtspunkten. Mit ihrer Hilfe kann eine inhaltsanalytisch gestützte Kartierung auch breit gesteckter Felder erfolgen. Beide Formen von Forschungssynthesen sind relevant für die Ableitung evidenzbasierter Entscheidungen in Wissenschaft, Politik 
und Praxis. Aufbauend auf einer derartigen Kartierung des Feldes ist es in darauffolgenden Arbeiten möglich, die empirische Evidenz zu spezifischen Fragestellungen metaanalytisch in aggregierenden Forschungssynthesen aufzubereiten. Insgesamt erleichtert dies Entscheidungsträgern in allen gesellschaftlichen Bereichen, sich ein Bild der verfügbaren Evidenz und ihrer Grenzen zu verschaffen und so informierte Entscheidungen zu treffen.

Eine systematische Suche nach Forschungssynthesen mit explizitem oder implizitem Bezug zur Digitalisierung in der kulturellen Bildung ergab keinen Hinweis auf Arbeiten, welche systematisch die relevante Forschung aus der Perspektive der kulturellen Bildungsforschung kategorisieren (Penthin et al. 2018). Stattdessen gerät kulturelle Bildung in vorliegenden Synthesen allenfalls am Rande von Überblicksarbeiten zu Anwendungsgebieten digitaler Technik in den Blick, unter anderem zu Videospielen, sozialen Netzwerken oder Suchmaschinen. Ein Beispiel dafür ist die Forschungssynthese von Lavranos et al. (2016) mit dem Titel „Information seeking for musical creativity: A systematic literature review" zur unterstützenden Nutzung digitaler Suchmaschinen für musikalisch-kreative Aktivitäten wie Komposition, Improvisation und das Analysieren von Musik. Daher wird in der vorliegenden Arbeit eine konfigurierende Forschungssynthese (Scoping Review) angestrebt, die auf Arbeiten basiert, welche Phänomene der Digitalisierung in der kulturellen Bildung explizit aus dem Blickwinkel der kulturellen Bildung betrachten. Dafür sollen die aus einer Datenbankrecherche resultierenden Ergebnisse zunächst auf ihre Relevanz hin beurteilt und anschließend gegebenenfalls qualitativ-inhaltsanalytisch nach bibliographischen, inhaltlichen und methodischen Gesichtspunkten kategorisiert werden.

\section{Forschungsfragen}

1. Wie verteilen sich die international vorliegenden Arbeiten zur Digitalisierung in der kulturellen Bildung nach den bibliometrischen Merkmalen (a) Art der Publikation (Zeitschriftenartikel; Konferenzbeitrage; Buch/Monographie, Buchkapitel und Sammelwerksbeitrag), (b) Affiliationsland der Erstautoren und (c) Publikationsjahr?

2. Wie verteilen sich die Arbeiten nach methodischen Bezügen (quantitativ-, qualitativ- oder gemischt-empirisch, sowie nicht-empirisch)?

3. Wie lassen sich speziell die empirischen Arbeiten jeweils nach folgenden untersuchten inhaltlichen Merkmalen kategorisieren:

a) Lebensphase (Kindheit, Jugend, Erwachsenenalter)?

b) Grad der Formalisierung (formal, non-formal, informell)?

c) Genres (Musik, Literatur, darstellende Künste inkl. Film und bildende Künste inkl. Fotografie)?

d) Art des digitalen Bezugs der untersuchten Phänomene

(Digitalisierungsgeneration 1, 2 und 3)?

4. Welche zentralen Fragestellungen lassen sich in den empirischen Arbeiten identifizieren und welche Evidenz liegt dazu vor? Welche Erträge ergeben sich daraus für die kulturelle Bildung? 


\section{Methode}

Basierend auf den theoretischen Überlegungen wurde (1) ein Suchbefehl für die Datenbankrecherche entwickelt, dieser wurde (2) angewendet auf die Datenbanken Fachportal Pädagogik und Scopus. Anschließend erfolgte (3) eine sukzessive Sichtung der Suchergebnisse zuerst nach Titeln, dann soweit verfügbar nach Abstracts und schließlich nach Volltexten. Für alle beibehaltenen Arbeiten folgte (4) die Kategorisierung nach bibliographischen, methodischen und inhaltlichen Gesichtspunkten. Die Vorgehensweise ist in Abb. 1 graphisch veranschaulicht. Schließlich wurden Beispiele für die Veranschaulichung prototypischer Forschungsfragen zu zentralen Facetten des Kategoriensystems ermittelt und dargestellt.

\subsection{Entwicklung des Suchbefehls}

Der Suchbefehl in der vorliegenden Arbeit beinhaltete 32 Begriffe für das Merkmal Digitalität (Details bei Christ et al. 2019). Diese wurden zum Teil in der ursprünglichen Fassung beibehalten (z. B. app, internet, computer), zum Teil wurden sie für die aktuelle Suche nach deutschsprachigen Arbeiten übersetzt (z. B. Medien, soziale Netzwerke) und als Oder-Verknüpfung integriert.

Für das Merkmal ,kulturelle Bildung“ wurden die folgenden im Feld gängigen Begriffe mit Oder-Verknüpfungen verwendet (vgl. Ijdens 2016; Keuchel 2012): ,kulturelle Bildung“, ,,ästhetische Bildung“ und ,,ästhetische Praxis“ für deutschsprachige Originalarbeiten, sowie ,cultural education“, ,,arts education“ und ,,aesthetic education" für internationale Originalarbeiten, die aus der Perspektive der kulturellen

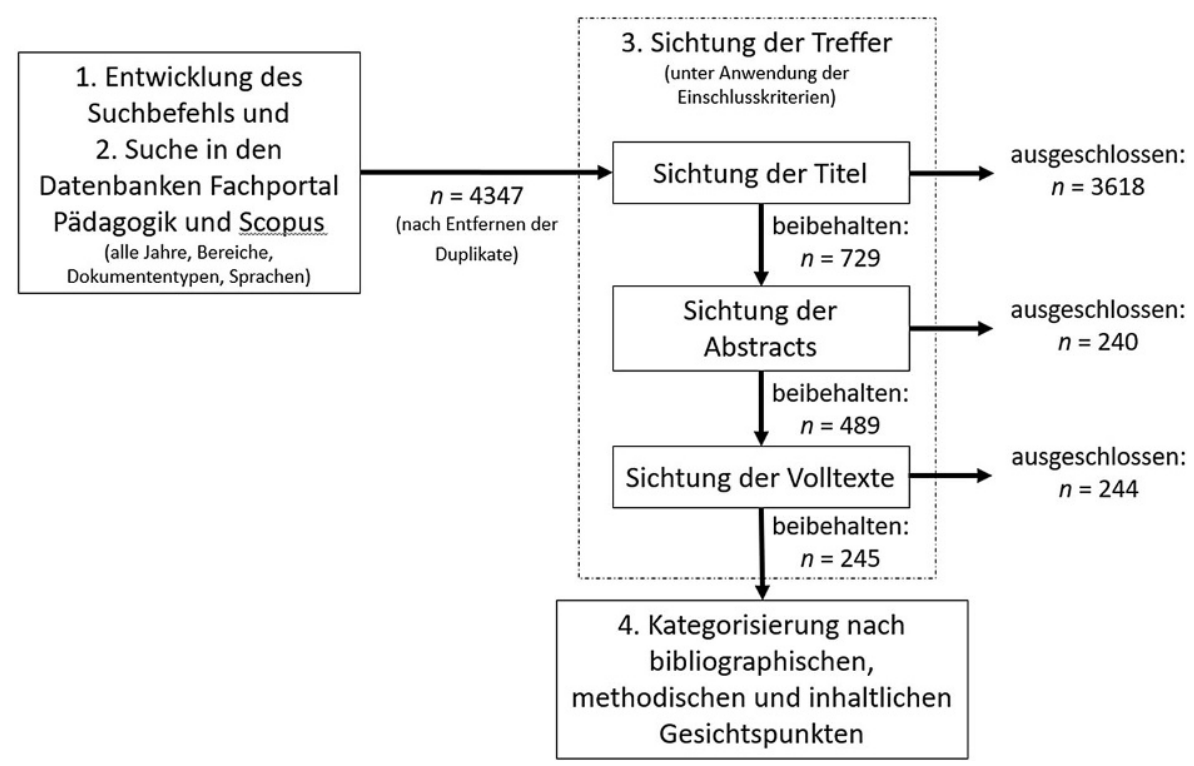

Abb. 1 Flussdiagramm des Recherche-, Sichtungs- und Auswertungsprozesses 
Bildung Phänomene an der Schnittstelle von Digitalisierung und kultureller Bildung in den Blick nehmen.

Für das Merkmal ,empirische Forschungsmethoden“wurden zunächst keine Einschränkungen durch den Suchbefehl vorgenommen. Stattdessen wurden sämtliche eingeschlossenen Publikationen nach Vorhandensein und ggf. Art des empirischen Bezugs kategorisiert. Analog dazu wurden auch keine Einschränkungen bzgl. bibliographischer Merkmale wie Publikationsjahr, Dokumententyp oder Fachdisziplin vorgenommen.

\subsection{Suche in Datenbanken}

Die Suche erfolgte im April 2019 in zwei großen Datenbanken: (1) im Fachportal Pädagogik, welches überwiegend bildungsbezogene Titel enthält und welche alle Einträge der vorwiegend deutschsprachigen Datenbank FIS-Bildung ebenso umfasst wie diejenigen aus den internationalen Datenbanken ERIC, Library of Congress, EBSCOhost ebooks, Casalini Libri und BBF 1945-1993; (2) in Scopus, einer der größten internationalen wissenschaftlichen Literaturdatenbanken. Die aus den beiden Suchen resultierenden Arbeiten wurden in einer Datenbank in erster Normalform mit allen notwendigen bibliographischen Angaben sowie Abstracts zusammengeführt, Dopplungen wurden entfernt. Bei Arbeiten ohne eingetragenen Abstract wurde dieser soweit vorhanden und zugänglich via Internetrecherche ergänzt.

Suchergebnisse: Aus der Anwendung des Suchbefehls auf die Datenbank des Fachportals Pädagogik resultierten $n=3849$ Treffer, auf Scopus $n=946$. Beide Trefferlisten wurden mit allen notwendigen bibliographischen Angaben einschließlich Abstracts exportiert und anschließend zusammengefügt. Nach Entfernung von $n=448$ Duplikaten resultierten insgesamt $n=4347$ Arbeiten.

\subsection{Sichtung der Treffer unter Anwendung der Einschlusskriterien}

Titel, Abstracts und Volltexte der aus der Suche resultierenden Arbeiten wurden der Reihe nach durch Mitglieder der Projektgruppe gesichtet (vgl. Abb. 1). Es wurden alle Arbeiten für den jeweils nächsten Sichtungsschritt ausgeschlossen, welche die Einschlusskriterien erkennbar nicht erfüllten.

Einschlusskriterien: Alle Arbeiten, die unserer Definition von digitaler kultureller Bildung entsprachen, wurden für die nachfolgende Kategorisierung nach bibliographischen Merkmalen und methodischen Bezügen berücksichtigt. Erst bei den weiterführenden Analysen nach inhaltlichen Kriterien erfolgte eine weitere Fokussierung auf die empirisch ausgerichteten Arbeiten, allerdings unabhängig davon, ob diese qualitative, quantitative oder gemischte empirische Bezüge aufwiesen. Zusätzlich mussten die berücksichtigten Arbeiten Originalarbeiten sein und einen englischen oder deutschen Volltext aufweisen. Arbeiten, die diese Kriterien nicht erfüllten, wurden ausgeschlossen. 


\subsection{Kategorisierung nach bibliographischen, methodischen und inhaltlichen Gesichtspunkten}

Bibliographisch erfolgte eine Kategorisierung nach Art der Publikation (Zeitschriftenartikel, Beitrag in Konferenzbänden, Buch/Monographie, Buchkapitel oder Sammelwerksbeitrag), Publikationsjahr und Affiliationsland des Erstautors. Für Zeitschriftenartikel wurde zudem der Zeitschriftentitel bestimmt.

Methodisch wurde differenziert in Arbeiten mit quantitativem, qualitativem oder gemischtem methodischem Bezug sowie in solche ohne empirischen Bezug (Jeong et al. 2014). Innerhalb der quantitativen Arbeiten wurde zudem wie eingangs erwähnt binnendifferenziert nach querschnittlichen, längsschnittlichen und Interventionsstudien (Bromme et al. 2014; Hussy et al. 2013). In Anlehnung an Jeong et al. (2014, S. 312 f.) haben wir für qualitative Arbeiten unterschieden in Arbeiten ohne explizite Bezüge auf eine verbreitete qualitativ-empirische Herangehensweise und solche, die derartige Bezüge aufweisen. Bei letzteren haben wir differenziert in Bezüge zu Grounded Theory, (qualitative) Inhaltsanalyse, Diskursanalyse, thematische Analyse/Kategorisierung und sonstigen Verfahren. Die darüber hinaus bei Jeong et al. (2014) benannten Ansätze wie Konversationsanalyse oder Interaktionsanalyse kamen in den eingeschlossenen Arbeiten nicht vor und wurden von uns daher im Kategoriensystem nicht separat berücksichtigt. Die Arbeiten mit gemischten methodischen Bezügen wurden differenziert danach, ob eine Strategie zur Kombination der methodischen Bezüge erkennbar war, und welche dies ggf. war (Creswell 2014).

Für die weitere inhaltliche Kategorisierung wurde auf die Teilmenge der empirischen Arbeiten fokussiert. Diese wurden nach dem Formalisierungsgrad des untersuchten Bildungsprozesses und der Lebensphase der Probanden kategorisiert. Ausschlaggebend dafür war, wo die in der jeweiligen Studie untersuchte Aktivität stattfand, nicht jedoch, ob die Datenerhebung an sich im formalen, non-formalen oder informellen Kontext durchgeführt wurde. Zudem folgte eine Einordnung nach Genre und Digitalisierungsgeneration. In Bezug auf das Genre wurden die Arbeiten entweder als genreübergreifend kategorisiert, oder sie wurden einer oder maximal zwei der vier Kategorien bildende Kunst (inklusive Fotografie), darstellende Kunst (inklusive Film), Musik oder Literatur zugeordnet. Hinsichtlich des digitalen Bezugs wurden die Studien anhand der jeweils thematisierten digitalen Werkzeuge einer von drei Digitalisierungsgenerationen zugeordnet. Wie eingangs erörtert umfasst die Digitalisierungsgeneration 1 Stand-Alone-Software sowie Web 1.0 „Read-Only“Anwendungen, Digitalisierungsgeneration 2 bezieht sich auf soziale Netzwerke und andere Web 2.0-Anwendungen mit nutzergenerierten Inhalten, Digitalisierungsgeneration 3 schließlich bezieht sich auf Phänomene wie Digital Making sowie Virtual und Augmented Reality und auf weitere aktuelle Entwicklungen im Kontext von Web 3.0 bzw. „Executable-Web“-Anwendungen. Jenseits der formalen Kategorisierung nach kulturellen Genres und Digitalisierungsgenerationen betrachten wir, welche Fragestellungen sich in der Gesamtheit der eingeschlossenen Artikel als zentral erweisen und beurteilen jeweils die Qualität der dazu vorliegenden Evidenz. 


\section{Ergebnisse}

Im Folgenden gehen wir auf die Ergebnisse des Sichtungsprozesses und der Kategorisierung der dabei eingeschlossenen Arbeiten ein. Wie im Methodenteil dargelegt erfolgen die inhaltlichen Analysen anhand prototypischer Studien zu Phänomenen der Digitalisierung in der kulturellen Bildung. Eine vollständige Auflistung aller im Ergebnisteil referenzierten Arbeiten einschließlich deren Kategorisierung nach bibliographischen, methodischen und inhaltlichen Aspekten sowie ihrer zentralen Forschungsfragen findet sich als Tabelle unter Kröner et al. (2021). Soweit nicht im Theorie- oder Diskussionsteil thematisiert sind die eingeschlossenen Arbeiten nicht als Referenzen im Literaturverzeichnis dieser Arbeit aufgeführt.

\subsection{Ergebnisse des Sichtungsprozesses}

Von den $n=4347$ in den Suchergebnissen enthaltenen Arbeiten wurden $n=3618$ (83\%) im Rahmen der Titelsichtung als irrelevant ausgeschlossen. Im Zuge der anschließenden Sichtung der Abstracts aller verbleibenden $n=729$ Arbeiten wurden $n=240$ weitere Arbeiten ausgeschlossen. Gleiches geschah mit $n=244$ der verbleibenden $n=489$ Arbeiten nach Sichtung der Volltexte. Darunter befanden sich $n=17$, deren Volltext weder auf Deutsch noch auf Englisch verfasst war, sowie $n=146$ von $n=268$ Arbeiten, für die ein Volltext weder über die Datenbanken noch durch Anfrage bei den Autoren erhältlich war. Alle nachfolgenden Analysen - soweit sie nicht noch stärker allein auf die empirischen Arbeiten fokussieren - basieren daher auf den insgesamt $n=245$ verbleibenden Arbeiten.

\subsection{Ergebnisse der Kategorisierung nach bibliographischen, methodischen und inhaltlichen Gesichtspunkten}

\subsubsection{Analyse nach bibliographischen Gesichtspunkten}

Unter den eingeschlossenen Arbeiten befanden sich $n=137$ Zeitschriftenartikel, $n=50$ Bücher, Buchkapitel und Monographien und $n=57$ Konferenzbeiträge. Hinweise auf eine Qualitätssicherung durch Peer-Review fanden sich auf den Webseiten von 104 Zeitschriften, aus denen $n=121$ der $n=137$ eingeschlossenen Zeitschriftenartikel stammen, und auf den Webseiten oder Beitragsaufrufen zu den Tagungen von $n=51$ der $n=58$ eingeschlossenen Konferenzbeiträge. Wenn bei Konferenzbeiträgen weder im Beitragsaufruf noch auf den Webseiten der Konferenz oder im Konferenzband ein Hinweis auf ein Review-Verfahren enthalten war, wurde die Arbeit als nicht peer-reviewed kategorisiert. Definiert über die Affiliationsländer der Erstautoren der relevanten Arbeiten ergab sich, dass die meisten Arbeiten aus den USA $(n=67)$, Deutschland $(n=54)$ und China $(n=17)$ stammten (vgl. Tab. 1$)$.

\subsubsection{Analyse nach methodischen Bezügen}

Von den $n=111$ empirischen Arbeiten ergab sich für $n=29$ eine quantitativ-empirische methodische Ausrichtung, für $n=60$ eine qualitativ-empirische und für 
Tab. 1 Eingeschlossene Publikationen nach Affiliationsländern

\begin{tabular}{lll}
\hline Rang & Affiliationsland von Erstautorin bzw. Erstautor & $\begin{array}{c}\text { Anzahl Publi- } \\
\text { kationen }\end{array}$ \\
\hline 1 & USA & 67 \\
2 & Deutschland & 54 \\
3 & China & 17 \\
4 & Australien & 12 \\
5 & Kanada & 10 \\
6 & Großbritannien & 9 \\
7 & Griechenland, Taiwan & 7 \\
8 & Finnland, Portugal & 6 \\
9 & Japan, Südkorea & 5 \\
10 & Kroatien, Schweden, Türkei & 4 \\
11 & Israel & 3 \\
12 & Ägypten, Niederlande, Dänemark, Österreich, Russland, Spanien, Tsche- & 2 \\
& chien & \\
& Algerien, Argentinien, Brasilien, Malaysia, Neuseeland, Ruanda, Singa- & 1 \\
\hline
\end{tabular}

$n=22$ ergaben sich gemischte methodische Bezüge. Ein Großteil dieser Arbeiten hat ein Peer-Review durchlaufen: Für quantitative Arbeiten waren dies $n=24$ von $n=29$, für qualitative $n=53$ von $n=60$ und für Studien mit gemischten Bezügen alle $n=22$ Arbeiten.

Differenzierte Betrachtung der quantitativen Arbeiten nach Studiendesigns In $n=12$ der $n=29$ eingeschlossenen quantitativ-empirischen Arbeiten wurden die Ergebnisse nicht inferenzstatistisch abgesichert. Zugleich wurde in keiner dieser Arbeiten Effektstärken berichtet, auch nicht in Form von Korrelationen. Vielmehr wurden lediglich deskriptive Statistiken wie Mittelwerte und Standardabweichungen berichtet. Diese wurden in $n=6$ Arbeiten genutzt, um Charakteristika einer Stichprobe zu einem Messzeitpunkt zu beschreiben, in $n=5$ Arbeiten um Gruppenunterschiede zu einem Messzeitpunkt zu illustrieren und in $n=2$ Arbeiten zur Ergebnisdarstellung von Längsschnittstudien.

Bei $n=17$ der $n=29$ eingeschlossenen quantitativ-empirischen Arbeiten wurden die Ergebnisse inferenzstatistisch abgesichert. Nur in $n=8$ dieser Arbeiten wurden Effektstärken berichtet. Die Studiendesigns der Arbeiten mit inferenzstatistischen Analysen ließen sich wie folgt unterteilen: Es waren $n=5$ querschnittlich ( 3 mit Effektstärken) und $n=2$ längsschnittlich ausgerichtet (davon 0 mit Effektstärken); $n=10$ wiesen ein quasiexperimentelles ( $n=7$, davon 2 mit Effektstärken) oder experimentelles Interventionsdesign auf ( $n=3$, davon 2 mit Effektstärken).

\section{Differenzierte Betrachtung der qualitativen Arbeiten nach Analysemethoden} und Studiendesigns In $n=42$ der Arbeiten, die qualitative Methoden verwendeten, wurden die methodischen Bezüge bei der Auswertung nicht ausdrücklich benannt. Vielmehr wurden lediglich Interviewausschnitte für die Illustrierung von Ergebnissen herangezogen oder es wurden für wesentlich erachtete Inhalte narrativ 
wiedergegeben. In den qualitativen Arbeiten mit expliziten methodischen Bezügen $(n=18)$ wurden Kodierverfahren am häufigsten verwendet (thematische Analyse/Kategorisierung, $n=6$, Grounded Theory, $n=4$ oder qualitative Inhaltsanalyse, $n=3$ ). Darüber hinaus gab es $n=2$ Arbeiten, die diskursanalytische Verfahren benutzten, eine Arbeit mit Auswertungen auf Grundlage der dokumentarischen Methode, und $n=2$ Arbeiten mit Bezügen zu sonstigen etablierten qualitativ-methodischen Verfahren. In eine übergeordnete Forschungsstrategie waren die Analysen von $n=6$ Arbeiten eingebettet, davon $n=4$ in partizipative Handlungsforschung und $n=2$ in entwicklungsbasierte Forschung (Design-Based-Research, DBR).

\section{Differenzierte Betrachtung der methodisch-gemischten Arbeiten nach Analyse-} methoden und Untersuchungsdesigns In $n=22$ Arbeiten wurden sowohl quantitative als auch qualitative Analyseverfahren verwendet. Bei $n=10$ dieser Arbeiten standen quantitative und qualitative Analysen nebeneinander, ohne für die Beantwortung einer Forschungsfrage kombiniert zu werden. Bei den restlichen $n=12 \mathrm{Ar}-$ beiten wurden für die Beantwortung zumindest einer Forschungsfrage qualitative und quantitative Daten miteinander kombiniert. Von diesen Arbeiten begründeten $n=3$ die verwendete Strategie anhand einschlägiger Literatur.

Im Rahmen der quantitativen Analysen wurden in den Studien mit gemischten methodischen Bezügen häufig weder Ergebnisse inferenzstatistischer Analysen noch Effektstärken berichtet $(n=15)$. Dies war lediglich bei $n=7$ Arbeiten der Fall, davon waren $n=1$ querschnittlich und $n=3$ längsschnittlich. Drei Arbeiten wiesen ein quasi-experimentelles $(n=2)$ oder experimentelles $(n=1)$ Interventionsdesign auf. Lediglich in einer der Arbeiten mit inferenzstatistischen Analysen wurde ein Effektstärkemaß berichtet.

Im Rahmen der qualitativen Analysen wurde in $n=11$ der Arbeiten mit gemischten Bezügen keine explizite Zuordnung zu verbreiteten Auswertungsverfahren vorgenommen. Unter den $n=11$ Arbeiten mit einer derartigen Zuordnung befanden sich $n=5$, die sich auf qualitativ-inhaltsanalytische Kodierverfahren beriefen und $n=5$, die thematische Analysen bzw. Kategorisierungsverfahren verwendeten. Hinzu kam eine auf die Grounded Theory bezogene Arbeit. Diskursanalysen oder sonstige Verfahren wurden in keiner Arbeit zu Grunde gelegt.

Publikationen pro Jahr nach empirischem Bezug Abb. 2 zeigt den Zuwachs der Zahl nicht-empirischer wie empirischer Publikationen zur Digitalisierung in der kulturellen Bildung über die Jahre hinweg. Die für nicht-empirische Arbeiten bereits ab dem Jahr 2006 feststellbare deutliche Erhöhung des jährlichen Publikationsaufkommens trat für empirische Arbeiten mit sechs Jahren Verzögerung auf.

\subsubsection{Analyse nach inhaltichen Gesichtspunkten}

Analyse nach Lebensphase und Grad der Formalisierung Im Rahmen der Analyse der $n=111$ empirischen Arbeiten nach inhaltlichen Gesichtspunkten erfolgte eine Kategorisierung nach Formalisierungsgrad sowie nach Lebensphase der Probanden. Der Großteil der Arbeiten basierte auf Daten von Probanden im Erwachsenenalter $(n=48)$, die meisten dieser Studien waren im Kontext von Hochschulen 


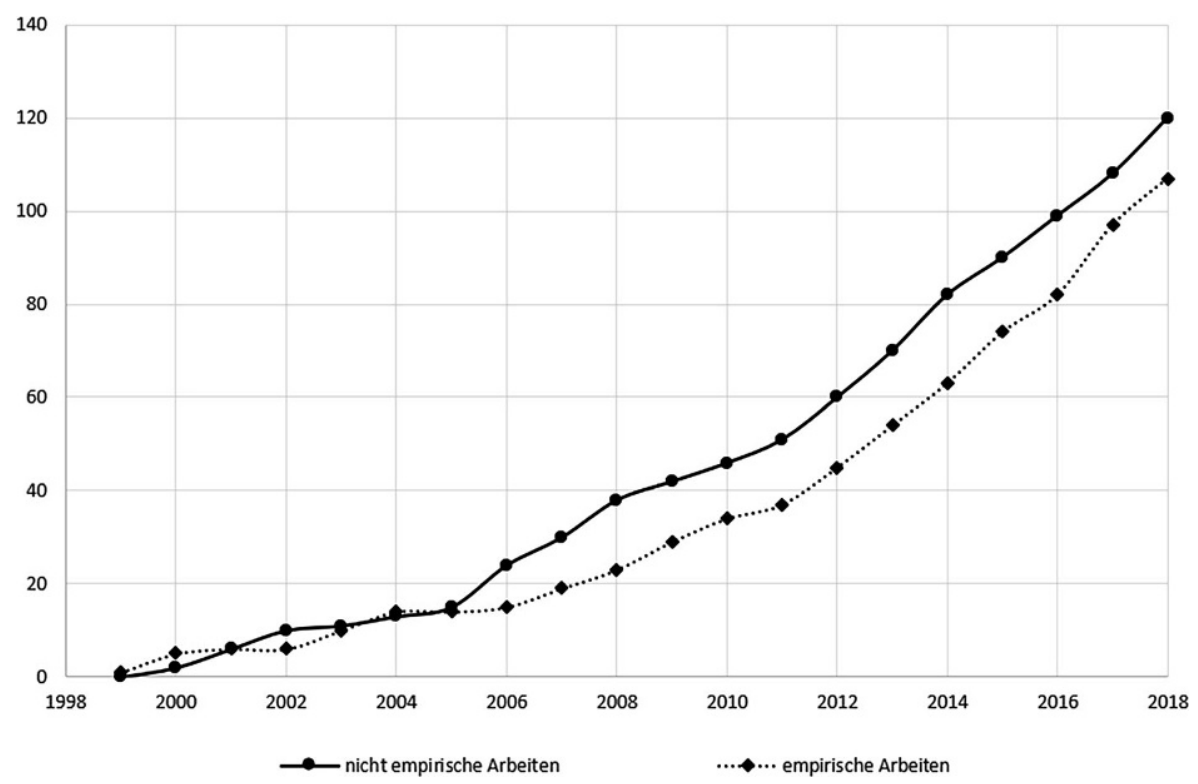

Abb. 2 Kumulative Anzahl empirischer und nicht empirischer Publikationen zur Digitalisierung in der Kulturellen Bildung nach Jahren im Zeitraum von 1998 bis 2018

angesiedelt. Daten von Jugendlichen wurden in $n=23$ Studien analysiert und solche von Kindern in $n=16$ Arbeiten. In $n=24$ Arbeiten umfasste die Stichprobe Probanden aus verschiedenen Lebensphasen.

Bei der Kategorisierung nach Formalisierungsgraden der Bildungsprozesse war für $n=13$ Arbeiten eine Mehrfachkodierung erforderlich (z. B. Burn 2016; Godau und Krebs 2017). Insgesamt $n=71$ Arbeiten konnten eindeutig formalen Kontexten zugeordnet werden, und $n=25$ non-formalen Kontexten. Es fanden sich lediglich $n=2$ Studien, die ausschließlich Bezüge auf informelle Kontexte aufwiesen (Geimer 2012; Gomes et al. 2014; vgl. Abb. 3).

Analyse nach Genre und digitalem Bezug Von den $n=79$ Arbeiten, die einem oder maximal zwei Genres zugeordnet werden konnten, bezogen sich $n=62$ auf die Kategorie bildende Kunst inklusive Photographie. Prototypische Beispiele für Forschungsanliegen (jeweils mit Bezug zu den einzelnen Digitalisierungsgenerationen) sind dabei: für die erste Generation die Arbeit zum Fotografieren mit einer App, die gleichzeitig Front- und Rückkamera des Smartphones aufzeichnet, mit dem Ziel, Anwendungsmöglichkeiten solcher Geräte für die Förderung kreativer Bildungsprozesse auszuloten (Güldenpfennig et al. 2014); für die zweite Generation eine Arbeit zum gemeinschaftlichen und eigenständigen Entwicklung von Videospielen mit einem Fokus darauf, inwieweit Kreativität bei derartigen Entwicklungsaktivitäten involviert ist und wie Schülergruppen die Arbeitsteilung bei Planung, visuellem Design und Programmierung organisieren (Sheridan et al. 2013); für die dritte Generation die Arbeit von Sun et al. (2011) zur Entwicklung eines 3D-VR-Programms zum Skizzieren und Malen und der Untersuchung der Frage, inwieweit es in schu- 


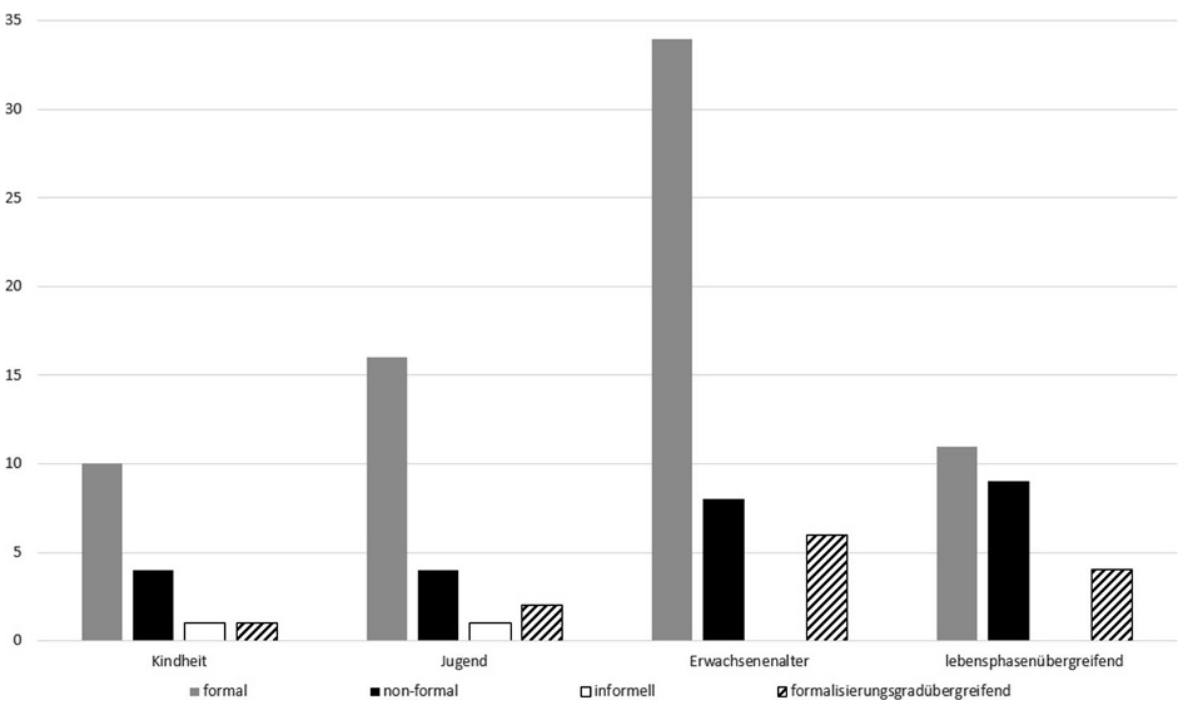

Abb. 3 Empirische Arbeiten zur Digitalisierung in der Kulturellen Bildung nach Formalisierungsgrad sowie nach Lebensphase der untersuchten Personen

lischen Angeboten zur bildenden Kunst verwendet werden kann und dabei positive Effekte auf den Lernerfolg bewirkt.

Im Bereich darstellende Kunst inklusive Film konnten 11 Arbeiten verortet werden. Beispiele sind hier: für die erste Digitalisierungsgeneration die Arbeit zum Einfluss der Verwendung bestimmter digitaler Design-Werkzeuge auf soziale Interessen von Kindern im Programm „Moviestorm“ im Rahmen der Erstellung eines eigenproduzierten Films (Burn 2016); für die zweite Digitalisierungsgeneration die Studie von Aubrey (2015) zur mittels sozialer Netzwerke ermöglichten gemeinsamen Erstellung eines Theaterstücks durch Kinder und Jugendliche in verschiedenen Ländern, in denen diese die Rolle von Angestellten einer Computerfirma spielen, und dessen anschließende Aufführung. Dabei wurden Effekte auf Weltoffenheit, Zusammenarbeit und interkulturelles Verständnis der Teilnehmenden bestimmt. Ein Beispiel für Digitalisierungsgeneration 3 ist die Untersuchung von Jeon (2017) zum Erleben von Kindern bei der Inszenierung und Aufführung eines Theaterstücks mit von ihnen programmierten Robotern.

Dem Bereich Musik wurden $n=10$ Arbeiten zugeordnet. Prototypisch sind hierzu: für die erste Digitalisierungsgeneration die Untersuchung von Wallerstedt (2013) zu Kommunikationsprozessen von Kindern über Musik, welche die Kinder mit einem auf ihre Keyboard-Eingaben reagierenden Programm selbst erzeugen; für die zweite Digitalisierungsgeneration eine Intervention zur Anwendung des Raspberry Pi und zugehöriger Sensoren für die Steigerung der Motivation zum aktiven, gemeinschaftlichen Musizieren von Grundschulkindern sowie der damit einhergehenden Vermittlung von Programmierkenntnissen (Sáez-López und Sevillano-García 2017). Für die dritte Digitalisierungsgeneration wurde hier erneut die für darstellende Kunst kodierte, bereits erwähnte Untersuchung von Jeon (2017) zu Theateraufführungen 
Tab. 2 Empirische Arbeiten nach Zugehörigkeit zu den Digitalisierungsgenerationen

\begin{tabular}{|c|c|c|c|c|}
\hline & \multicolumn{3}{|c|}{ Digitalisierungsgeneration } & \multirow[b]{2}{*}{ Insgesam } \\
\hline & 1 & 2 & 3 & \\
\hline Bildende Kunst & 38 & 19 & 5 & 62 \\
\hline Darstellende Kunst & 5 & 5 & 1 & 11 \\
\hline Musik & 6 & 3 & 1 & 10 \\
\hline Literatur & 4 & 2 & 0 & 6 \\
\hline Genreübergreifend & 8 & 15 & 9 & 32 \\
\hline Summe & 61 & 44 & 16 & $122^{\mathrm{a}}$ \\
\hline
\end{tabular}

${ }^{\mathrm{a} A u f g r u n d}$ der Kodierung von $n=10$ Arbeiten in zwei Genres ist die Gesamtzahl höher als die Anzahl zugrundeliegender empirischer Arbeiten.

mit programmierten Robotern kodiert, da die Kinder auch die Musik und Klangeffekte zum Theaterstück selbst komponiert bzw. programmiert haben.

Für den Bereich Literatur waren $n=6$ Arbeiten verfügbar; z. B. zur ersten Digitalisierungsgeneration die Arbeit von Reimann (2008) zum Schreiben fiktiver Geschichten durch Kinder und deren visuelle Gestaltung durch Hypermedia im Kunstunterricht mit dem Ziel der Förderung von Media Literacy; zur zweiten Digitalisierungsgeneration die Arbeit von Bucur (2006) dazu, wie eine e-LearningUmgebung dazu beitragen kann, kreative Schreibkompetenzen zu fördern. Zur dritten Digitalisierungsgeneration waren hier keine Arbeiten auffindbar.

Insgesamt $n=32$ empirische Arbeiten erwiesen sich als genreübergreifend. Als prototypisch für die einzelnen Digitalisierungsgenerationen lassen sich hier nennen: Für die erste Generation die Arbeit von Paliokas und Kekkeris (2008) zur Entwicklung eines webbasierten 3D-Museums und die Erprobung seiner Anwendung im Kunstunterricht in der Primar- und Sekundarstufe mit dem Ziel, das Interface und die Navigation in dem Prototypen zu verbessern; für die zweite Generation die Arbeit zur Förderung des Verständnisses der jeweils anderen Kultur bei amerikanischen und türkischen Kindern durch Erstellung von digitalen Präsentationen über die eigene Kultur und ihren Austausch über soziale Netzwerke von Simsek und Nuss (2010); für die dritte Generation die Arbeit zum kreativen, interaktiven Spielen einer hawaiianischen Legende in einer VR-Umgebung von Ge et al. (2003).

Wie Tab. 2 zeigt, war in Bezug auf die Digitalisierungsgenerationen die am stärksten besetzte Kategorie die der Arbeiten zu Computerprogrammen wie Apps oder Videospielen ohne soziale Vernetzung (erste Digitalisierungsgeneration). Immer noch recht häufig waren Arbeiten zu digitalen Techniken, die soziale Vernetzung ermöglichen oder Zusammenarbeit erfordern (zweite Digitalisierungsgeneration). Deutlich seltener waren im Vergleich dazu Arbeiten zu Technologien aus dem Bereich Virtual und Augmented Reality oder Digital Making (dritte Digitalisierungsgeneration).

\subsubsection{Analyse der zentralen Fragestellungen und vorliegender Evidenz}

Wie lauten die im Rahmen der einzelnen Genres untersuchten zentralen Fragestellungen und welche Qualität weist die vorliegende Evidenz auf? Im Folgenden werden die untersuchten Fragestellungen gruppiert nach Genres dargestellt. Soweit innerhalb des Genres thematische Schwerpunkte und Unterschwerpunkte identifi- 
ziert wurden, wird gegebenenfalls noch weiter danach untergliedert. Innerhalb der Genres oder ggf. Schwerpunkte werden dann jeweils im Detail zunächst von den quantitativen Studien diejenigen dargestellt, die einen hohen Evidenzgrad aufweisen, indem sie auf der Basis von (quasi-)experimentellen Designs Erklärungs- und Veränderungswissen bereitstellen (Bromme et al. 2014; Hussy et al. 2013). Es folgt eine summarische Auflistung der Studien mit schwächerer quantitativer Evidenz. Last but not least werden Studien mit qualitativen Elementen vorgestellt, da diese gerade in sich entwickelnden Forschungsgebieten oft inspirierende Beiträge leisten. Weitere $n=22$ Studien, die keinem der identifizierten Schwerpunkte zugeordnet werden konnten, darunter lediglich eine mit zumindest quasi-experimentellem Design, finden sich in der Tabelle aller eingeschlossenen Studien bei Kröner et al. (2021).

Arbeiten zum Genre der bildenden Künste Unter den $n=62$ Arbeiten zum Genre der bildenden Künste ergaben sich drei Schwerpunkte: (1) Studien, die ausschließlich formale Bildungsangebote im Bereich der bildenden Künste untersucht haben $(n=35)$; (2) Arbeiten, die den Einsatz digitaler Werkzeuge in interdisziplinären oder außerkünstlerischen formalen Bildungsangeboten untersucht haben $(n=10)$; (3) Arbeiten zum Einsatz digitaler Werkzeuge bei der Förderung künstlerischer Aktivitäten in non-formalen Bildungsangeboten $(n=2)$. Die $n=35$ Arbeiten innerhalb des erstgenannten Schwerpunkts zu formalen Bildungsangeboten der bildenden Künste lassen sich nochmals untergliedern in Unterschwerpunkte zu (1.1) Kompetenzerwerb, Motivation und Interesse $(n=29)$ einerseits und $\mathrm{zu}(1.2)$ digitalem Peer-Feedback $(n=3)$ andererseits.

(1.1) Kompetenzerwerb, Motivation und Interesse in formalen Angeboten zu den bildenden Künsten: Unter den $n=29$ Arbeiten dieses Unterschwerpunkts thematisieren zwei (quasi-)experimentelle Arbeiten den Effekt des Einsatzes von VR-Brillen im Unterricht auf Kriterien wie das Zeichnen von 2D- und 3D-Objekten oder die Initiierung selbständiger Lernprozesse (Bolier et al. 2018; Sun et al. 2011). Bei grundsätzlich positiven Effekten war der Effekt auf Motivation, Interesse und Spaß an der Tätigkeit generell deutlich ausgeprägter als der Effekt auf den Kompetenzerwerb, der sich zudem nicht immer statistisch absichern ließ. Auch experimentelle Studien zu gamifizierten Malprogrammen ergaben positive Effekte auf den Erwerb künstlerischer Kompetenzen (Tomljenovic 2015). Zu den experimentellen Befunden analoge Ergebnisse finden sich auch in einer Vielzahl $(n=12)$ von quantitativen Studien mit schwächeren Designs (Busby et al. 2000; Cutcher und Cook 2016; Drosos et al. 2018; Ho und Lin 2015; Ji et al. 2009; Liao und Ho 2011; Mohamad 2011; Peppler 2010; Prohaska 2012; Ueno et al. 2013; Vuk et al. 2015; Wang und Lv 2018): Der Effekt digitaler Werkzeuge ist - allerdings Studien ohne inferenzstatistische Analysen zufolge - insbesondere dann stärker ausgeprägt, wenn Gamification stattfindet und die Aktivität Elemente eines Videospiels wie Punkte-Highscores aufweist (Price 2007; Ueno et al. 2013; Wang und Lv 2018; Williford et al. 2017).

Ein ähnliches Bild wie bei den quantitativen Studien ergibt sich auch für die $n=14$ Arbeiten mit qualitativen Elementen zum Einsatz digitaler Werkzeuge in formalen Bildungsangeboten zu den bildenden Künsten. In Aktivitäten wie der künstlerischen Gestaltung von Bühnen für Theaterstücke mit digitalen Werkzeugen (Loveless 2003; Reimann 2008; Richardson 2016), dem gemeinsamen Gestalten von di- 
gitalen Kunstwerken in Online-Plattformen (Black und Cap 2014; Thevenin 2012; Wimpenny et al. 2019) und dem Erstellen von animierten Kunstwerken für 3DUmgebungen (Betts 2003; Burn 2016; Lu 2013; Reimann und Biazus 2007; Zuiker 2014) zeigten die Schülerinnen, Schüler und Studierende ausgeprägte Kreativität, hohes Interesse und starke Motivation. Die Werke von den Teilnehmenden erstellten Werke bezogen sich häufig auf ihr persönliches Umfeld oder die jeweilige Jugendkultur (Castro 2014; Güldenpfennig et al. 2014; Richardson 2016; Rissanen 2017).

(1.2) Digitales Peer-Feedback in formalen Bildungsangeboten im Bereich der bildenden Künste: Unter den $n=3$ Arbeiten dieses Unterschwerpunkts befinden sich zwei quantitative Studien und eine Studie mit gemischten methodischen Bezügen.

Die einzige zu diesem Schwerpunkt vorliegende experimentelle Studie zeigte einen positiven Effekt von kriterienbasiertem Peer-Feedback auf die künstlerischen Kompetenzen (Chen et al. 2017). Einer weiteren Studie mit - mangels Kontrollgruppe - schwächerem Design zufolge führt Tablet-basiertes Peer-Feedback mit schwerhörigen Schülerinnen und Schülern zu einer Steigerung der künstlerischen Kompetenz und mehr Spaß am Lernprozess (Namatame und Matsuda 2012).

Qualitative Elemente finden sich in der methodisch gemischten Studie von Dikici (2009), in der basierend auf Interviews und Korrelationsanalysen untersucht wurde, wie verschiedene Ausprägungen digitalen Peer-Feedbacks im Kunstunterricht integriert werden können (Dikici 2009).

(2) Einsatz digitaler Werkzeuge in interdisziplinären oder außerkünstlerischen formalen Bildungsangeboten: Die $n=11$ Arbeiten zu diesem Schwerpunkt sind sämtlich qualitativ: Dufva (2018) untersuchte, wie das eigenständige Entwickeln, Gestalten, Bauen und Programmieren von interaktiven Kunstwerken im Informatikunterricht das Verstehen von Inhalten aus Informatik und Physik fördert. O'Gorman und Davis (2013) untersuchen, welche Lernprozesse durch Interventionen angestoBen werden, die sowohl die Nutzung eines Programms zur Berechnung des eigenen ökologischen Fußabdrucks als auch künstlerische Aktivitäten wie das Zeichnen von Selbstportraits umfassen. In mehreren Studien schlossen die Autoren, dass eine interdisziplinäre Verknüpfung von digitalen Werkzeugen mit künstlerischen Aktivitäten einen deutlichen Mehrwert für Kompetenzentwicklung, Interesse und Motivation mit sich bringe (Asher 2007; Einfeld 2018; Foreman-Wernet et al. 2014; Marner 2013; Marner und Örtegren 2013). Gleichzeitig wurde häufig angemerkt, dass digitale Werkzeuge ihr Potenzial nur dann voll ausschöpfen, wenn sie sinnvoll in die formalen Bildungsangebote integriert sind und nicht lediglich für die künstlerische Aktivität genutzt werden, sondern auch als digitale Werkzeuge für das Teilen der resultierenden Werke und den Austausch darüber (Einfeld 2018; Marner 2013;). Zusätzlich zeigte sich, dass Kinder kreativer mit digitalen Werkzeugen umgehen als Erwachsene, da diesen häufig das Selbstvertrauen bei der Verwendung von Computern fehlt (Dollhausen 2000; Heydon 2012). Ståhl und Kollegen (2018) empfehlen die Integration von Gamifizierung in formale Bildungsangebote, und zwar in einer Form, die sich nicht auf die Vergabe von Punkten und Abzeichen an Kinder beschränkt, sondern explizit künstlerische Komponenten umfasst, beispielsweise in Form der Möglichkeit zur individuellen Gestaltung von Avataren.

(3) Einsatz digitaler Werkzeuge bei der Förderung künstlerischer Aktivitäten in non-formalen Bildungsangeboten: $\mathrm{Zu}$ diesem Schwerpunkt lag eine quantitative und 
eine methodisch gemischte Arbeit vor, von denen jedoch keine ein (quasi-)experimentelles Design aufwies. Sowohl Jäckel und Reinhardt (2000), als auch Thiedeke (2000) fokussierten dabei auf die Erwachsenenbildung und konstatierten positive Effekte auf künstlerische Kompetenzen, Interesse und Aktivitätsmotivation.

Über alle Schwerpunkte innerhalb der bildenden Künste hinweg wurden als potenzielle Anwendungsbarrieren von digitalen Werkzeugen in künstlerischen Aktivitäten Vorbehalte gegenüber der künstlerischen Verwendung technischer Geräten (Jäckel und Reinhardt 2000; Mak und Degennaro 1999), geringe technische Kompetenzen auf Lehrerseite (Thiedeke 2000) und, im Fall von Fernunterricht, fehlende reale Treffen (Cutcher und Cook 2016) genannt. Die beiden erstgenannten Barrieren wurden jedoch in mehr als 20 Jahre alten Studien thematisiert, neuere Studien haben derartige Probleme nicht benannt.

Arbeiten zum Genre der darstellenden Kunst Mit $n=8$ von $n=11$ Studien ergab sich für Arbeiten zur darstellenden Kunst ein Schwerpunkt auf formale Bildungsangebote mit Bezug auf das Theater.

Für diesen Schwerpunkt, und für das Genre der darstellenden Kunst insgesamt, konnte nur eine experimentelle Studie identifiziert werden. Diese ergab einen positiven Effekt der Verwendung einer Online-Lernplattform im Theaterunterricht auf den Lernerfolg von Kindern (Griffin et al. 2009). Wie schon bei Bolier et al. (2018) und Sun et al. (2011) ließ sich der Effekt einer Verwendung von digitalen Plattformen auf den Lernerfolg nicht statistisch absichern, wohl aber ein Effekt auf das Interesse bzw. die Motivation, an zukünftigen Aufführungen der Schule als Regisseur, Autor oder Schauspieler mitzuwirken. Darüber hinaus legen quantitative Studien mit schwächeren Designs und ohne inferenzstatistische Absicherung nahe, dass Schülerinnen und Schüler durch ihre Einbindung in die Erstellung von Lernvideos und durch die Integration ihres Feedbacks an die Ersteller der Lernvideos einen höheren Lernerfolg und ein höheres Interesse aufweisen als durch simples Ansehen der Lernvideos (Teo und Chai 2009), und sie deuten an, dass Studierende aus dem Bereich Theater das Filmen und Teilen eigener Antworten mit Smartphones positiv bewerten (Li 2017).

Unter den $n=7$ qualitativen Arbeiten des Schwerpunkts formale Bildung und Theater befinden sich $n=5$ Studien, die auf eine Verwendung digitaler Werkzeuge im Theaterunterricht fokussierten. Untersucht wurde hier, wie Kinder digitale Schauspieler animieren (Burn 2016) können. Bae (2016) untersuchte, wie Kinder Videos für Peers in anderen Ländern filmen und mit diesen darüber digital kommunizieren. In der Studie von O'Toole und Dunn (2008) haben Schulkinder ein Theaterstück über ihre parallel laufende Entwicklung eines Lernprogramms im Geschichtsunterricht geplant, geschrieben und aufgeführt. Jeon (2017) untersuchte, wie Schülerinnen und Schüler eine 3D-Umgebung nutzen, um Musik und Bilder in Tanzbewegungen von Avataren zu verwandeln und umgekehrt gefilmte Tanzbewegungen in digitale Musik und Bilder. Wie in den entsprechenden quantitativen Studien werden auch hier positive Effekte auf Kreativität, Interesse und Motivation berichtet. Darüber hinaus deutet sich an, dass gezielter Unterricht nötig ist, damit Schulkinder von der Möglichkeit zur Verwendung digitaler Werkzeuge profitieren (Burn 2016) und dass 
deren Integration in den Theaterunterricht abhängig ist von schulischer Ausstattung und Kompetenz der Lehrkräfte (Loveless 2003).

Arbeiten zum Genre Musik Bei den Arbeiten zum Genre Musik ( $n=10)$ kristallisierte sich Musiklernen bzw. Musizieren mit digitalen Werkzeugen als Schwerpunkt heraus $(n=6)$. Hier lag mit der Arbeit von Sáez-López und Sevillano-García (2017) lediglich eine quasiexperimentelle Studie vor. Dort wurden Effekte einer digitalen Intervention auf Lernerfolg, Interesse und musikpraktischen Kompetenzen von Kindern mit Effekten klassischen Musikunterrichts verglichen. In der Intervention galt es, elektronische Gitarren zu gestalten und zu programmieren, die mit Hilfe von Sensoren und Einplatinencomputern (Raspberry Pi) zum Musizieren verwendet werden können. Wie schon bei Bolier et al. (2018), Sun et al. (2011) und Griffin et al. (2009) zeigte sich auch hier kein signifikanter Effekt auf musikpraktische Kompetenzen, wohl aber auf Interesse und Motivation - sowie in diesem Fall auf die Programmierkenntnisse. Des Weiteren untersuchten zwei quantitative Studien mit mangels Kontrollgruppe schwächerem Prä-Posttest-Design den Effekt der Nutzung von digitalen Musikprogrammen bzw. den digitalen Musizierens auf verschiedene Outcomes: Bei Yoo und Lee (2019) zeigte sich, dass nach sechs aufeinander aufbauenden Sitzungen mit dem Musikprogramm Sonic Pi in Hochschulkursen für Nicht-Musiker und Nicht-Künstler deren Kreativität, Interesse und Motivation höher war als vor den Sitzungen. Für die Anwendung des Musiklernspiels Flappy Crab mit 9 bis 12-Jährigen ergaben deskriptive Analysen durch Gomes et al. (2014) eine kontinuierliche Steigerung der musikalischen Kompetenz über eine Phase von 14 Wochen hinweg, begleitet von einer allgemeinen hohen Zufriedenheit und ausgeprägtem Spaß beim Spielen.

Schließlich fanden sich drei qualitative Arbeiten zum Schwerpunkt Musizieren bzw. Musiklernen mit digitalen Werkzeugen: Sander-Steinert (2016) untersuchte die gemeinschaftliche Verwendung von Tablets durch Schulkinder mit Förderbedarf für die digitale Verklanglichung einer Ballade. Lierse (2015) beschreibt die Vor- und Nachteile der Umstellung auf digitale Lehre im Bereich Musik an neun verschiedenen Standorten der australischen Charles-Darwin-Universität. Als Vorteil gibt sie an, dass mehr Studierende durch die digitale Lehre erreicht werden können, jedoch das gemeinsame Musizieren und Diskutieren in nicht-digitalen Gruppen als fehlend empfunden wird. Wallerstedt (2013) schließlich analysierte die Erfahrungen mit einem Programm, dass die gemeinschaftlich komponierte Musik analysiert, und zeigte, dass die von ihr untersuchten 7-jährigen Kinder im Laufe des Prozesses eigene Beschreibungen für die musikalischen Prozesse entwickeln.

Arbeiten zum Genre Literatur Im Genre Literatur $(n=6)$ ergab sich als Schwerpunkt - mit $n=5$, sämtlich qualitativen Studien - die Anwendung von digitalen Werkzeugen in gemeinschaftlichen Schreibprozessen. Dies wurde untersucht für das Schreiben von Theaterstücken (Reimann 2008), das Verfassen von Geschichten über bekannte Kunstwerke (Tanase et al. 2017), das Erstellen von Essays in der Hochschule (Bucur 2006), das Erarbeiten von digitalen Nachrichteninhalten zu lokalen Problemen und Herausforderungen (Thevenin 2012) und für das autobiographische Schreiben von Geflüchteten (Sawhney 2009). Positive Effekte auf Kreativität, Moti- 
vation und sozialen Kompetenzen (Bucur 2006; Reimann 2008; Tanase et al. 2017) deuteten sich ebenso an wie solche auf bürgerschaftliches Engagement (Thevenin 2012) und Empowerment der Teilnehmerinnen (Sawhney 2009).

Genreübergreifende Arbeiten Unter den Studien, die nicht einem oder maximal zwei Genres zugeordnet werden konnten $(n=32)$, ließen sich zwei Schwerpunkte identifizieren: (1) im Hinblick auf das Kriterium solche zu Effekten digitaler Lerneinheiten auf kulturelles Lernen ( $n=18)$; (2) im Hinblick auf das Setting solche zu virtuellen Ausstellungen, Führungen und Museen $(n=7)$.

(1) Kulturelles Lernen: Im Schwerpunkt kulturelles Lernen zeigten $n=4$ (quasi-)experimentelle Studien erneut positive Effekte digitaler Werkzeuge auf Lernerfolg und Lernmotivation, beispielsweise durch die Nutzung von Smartphones als im Alltag stets verfügbare und die Lebenswelt durchdringende Lernplattform für Studierende, beispielsweise anhand einer App zu Sehenswürdigkeiten in Taiwan (Chin et al. 2018). Positive motivationale Effekte ergaben sich, wenn eine digitale Lernplattform es Kindern ermöglicht, sich über gamifizierte Apps zum kulturellen Lernen Punkte zu verdienen, um den eigenen Avatar kreativ individualisieren zu können (Lin et al. 2018). Derartige Effekte auf interkulturelles Lernen deuteten sich auch in den Experimenten von Muir (2010) mit Wikis und eBoards im spanischen Fremdsprachenunterricht und von Stockwell (2016) mit WebQuests mit japanischen Studierenden in Kursen zu interkultureller Kommunikation an. Allerdings ließen sich in beiden Studien die deskriptiv vorhandenen positiven Effekte auf das Lernergebnis nicht inferenzstatistisch absichern (Muir 2010; Stockwell 2016), wohl aber zumindest bei Muir (2010) die auf Zufriedenheit und Lernmotivation. Dieses Muster positiver Effekte der digitalen Aktivität auf Lernerfolg, Lernmotivation und Zufriedenheit im Rahmen des kulturellen Lernens findet sich auch in den $n=6$ quantitativen Studien mit schwächeren Designs wieder (Chen et al. 2014; Gubanihina et al. 2017; Okubo und Kumahata 2001; Pappa et al. 2018; Shih 2015; Simsek und Nuss 2010).

Im Fokus qualitativer Studien zu kulturellem Lernen $(n=8)$ standen Blogging mit Mobiltelefonen (Shao und Crook 2015), digitale Kommunikationsplattformen (Dunn und Occhi 2003; Kutay et al. 2012; Magro et al. 2014; Reading et al. 2013), ein digitales Klassenzimmer (Ammaria und Azzeddine 2014; Derni und Bouhassoun 2015), selbstgestaltete Videos (Cornell 2012) und eine VR-Umgebung (Ge et al. 2003). In allen Studien werden das hohe Interesse und die hohe Kreativität der Teilnehmenden hervorgehoben, bei zugleich vorhandenen Herausforderungen wie technischen Problemen oder Sprachbarrieren bei der Kommunikation mit anderen international-verteilten Gruppen.

(2) Virtuelle Ausstellungen, Führungen und Museen: Zu diesem Schwerpunkt gehörten drei quantitative Studien, darunter keine (quasi-)experimentelle. Quantitativ untersucht wurden die Nutzung von VR-Museen in Schulen (Paliokas und Kekkeris 2008), VR-Touren durch London mit Google Street View (Shih 2015) oder Museumsführungs-Apps auf Smartphones (Kali et al. 2014). In allen drei Studien deutete sich deskriptiv ein positiver Effekt digital vermittelter Aktivitäten auf Zufriedenheit und Motivation der Teilnehmenden an. 
Es lagen $n=4$ qualitative Studien zu virtuellen Ausstellungen, Führungen und Museen vor: Clarke und Lewis (2016) untersuchten die Darstellung von kulturellen Identitäten von Personen benachteiligter Gruppen in digitalen Museen und wie diese von Besuchern durch das Teilen eigener Erfahrungen angereichert werden können. Durch diesen Prozess erfuhren alle Museumsbesucher der benachteiligten Gruppen Empowerment, nicht nur diejenigen, die ihre eigenen Erfahrungen teilen. Noch aktiver gestaltete sich die von Roussou et al. (2007) untersuchte Planung, Entwicklung, Gestaltung und Umsetzung eines digitalen Museums durch 11-jährige Freiwillige in Athen, die dabei ein sehr hohes Engagement und eine hohe Motivation zeigten. Hayhoe (2014) hat den Besuch realer und digitaler Museen mit verbalen Beschreibungen der Ausstellungsstücke für blinde Jugendliche untersucht. Diese haben trotz der fehlenden visuellen Komponente kognitive und emotionale Verbindungen zu den Ausstellungstücken aufgebaut. Shim und Park (2013) haben in einer Gamifizierungsstudie untersucht, wie sich insbesondere Kinder motivieren lassen, wenn sie individuelle, durch eigene Leistungen ,verdiente“ digitale Stempel an selbstgewählten Stellen in virtuellen Museen hinterlassen können.

Über alle Studien zur Implementation digitaler Museen und Museumsführungen hinweg wurde als Hindernis der generell hohe technische und zeitliche Aufwand ebenso benannt wie die fehlende Möglichkeit, die Teilnehmenden bei der Entwicklung der Ausstellungen zu integrieren. Allerdings sind diese Ergebnisse zurückhaltend zu interpretieren, da sie weder auf (quasi-)experimentellen Designs beruhten noch inferenzstatistisch abgesichert wurden.

\section{Diskussion}

\subsection{Zusammenfassung der Publikationslage und Implikationen}

Sprache, Genre, Digitalisierungsgeneration und Formalisierungsgrad: In Bezug auf das Genre nahm ein Großteil der identifizierten empirischen Arbeiten eine übergreifende Perspektive ein. Wenn auf ein spezifisches Genre fokussiert wurde, war es häufig das der bildenden Künste. Hinsichtlich des digitalen Bezugs ließen sich die Arbeiten meist in der ersten Digitalisierungsgeneration verorten. Deutlich weniger Arbeiten bezogen sich auf soziale Netzwerke und andere Phänomene der zweiten Digitalisierungsgeneration, und nochmals weniger Arbeiten auf Phänomene der dritten Digitalisierungsgeneration wie Digital Making oder Virtual Reality. Bezüglich des Formalisierungsgrades betreffen die meisten Arbeiten formale und non-formale Settings, und hinsichtlich der Lebensphase der Probanden liegt ihr Fokus entweder auf der Kindheit oder dem Jugendalter.

Methodische Bezüge: Mit kaum mehr als 100 Arbeiten ist der Umfang in einschlägigen Datenbanken identifizierter empirischer Arbeiten zur Digitalisierung in der kulturellen Bildung noch überschaubar (Projektgruppe „Forschung zur Kulturellen Bildung in Deutschland“ 2014). Deren überwiegende Mehrheit ist qualitativ-empirisch ausgerichtet. Dabei fällt unmittelbar ins Auge, dass sie oft nicht explizit auf bestimmte Herangehensweisen wie rekonstruktive Methoden oder die qualitative Inhaltsanalyse Bezug nehmen oder diese nicht systematisch anwenden. Häufig werden 
qualitative Daten lediglich zur Illustration einer im Fokus stehenden Beschreibung der Anwendung der digitalen Werkzeuge genutzt. Unter den quantitativen Arbeiten fanden sich nur wenige Interventionsstudien. Ein Großteil davon arbeitete nicht mit randomisierter Zuweisung der Probanden zu den Gruppen und randomisierter Bestimmung, welche Gruppe Kontroll- und welche Experimentalgruppe ist. Auch die wenigen vollwertigen Experimente oder Quasi-Experimente blieben in der Auswertung häufig aufgrund fehlender inferenzstatistischer Absicherung der Befunde sowie fehlenden Informationen über Effektstärken hinter den Möglichkeiten zurück. Ebenso waren - insbesondere bei Studien ohne Kontrollgruppe - die verwendeten quantitativen Methoden häufig sehr basal. Schon Korrelations- und Regressionsanalysen waren selten, Strukturgleichungsmodelle wurden erst recht nur in Einzelfällen angewendet, obwohl die vorhandene Datenlage dies häufig zugelassen hätte. Wie schon für die experimentellen Studien konstatiert, zeichnete sich auch über alle Studiendesigns hinweg ab, dass vergleichsweise selten Effektstärken berichtet wurden. Dies gilt auch dort, wo Signifikanztests zur statistischen Absicherung verwendet wurden. Auch die Chance zu einer Verschränkung quantitativer und qualitativer Herangehensweisen im Sinne von Mixed Methods wurde nur selten ergriffen, und wenn, dann wurde das darin liegende Potenzial nicht ausgeschöpft. Der DBR-Ansatz schließlich spielte bei den identifizierten Arbeiten bislang kaum eine Rolle, obwohl er sich in besonderer Weise für eine anwendungsnahe Forschung zur Entwicklung von digitalen kulturellen Bildungsangeboten eignen würde. Insgesamt erscheint es daher lohnend, die vorliegenden Datensätze vertieft auszuwerten, um ihr Potenzial zur Bereitstellung von Evidenz zu nutzen.

Inhaltliche Forschungsschwerpunkte: Inhaltlich wurde über alle Genres hinweg häufig untersucht, wie kognitive und motivationale Ergebnisse von Bildungsprozessen durch verschiedene digitale Werkzeuge beeinflusst werden. Ein Großteil dieser Studien bezog sich auf Effekte der Digitalisierung formaler Bildungsangebote im Bereich Kunst und Theater, interdisziplinäre Studien zu anderen Genres waren deutlich seltener. Auf der Kriteriumsseite lag der Fokus meist auf Lernerfolg, Kompetenzerwerb, Interesse und Motivation. Darüber hinausgehende Effekte auf Persönlichkeitsentwicklung oder Empowerment wurden seltener in den Blick genommen. Unabhängig vom verwendeten Kriterium generell selten waren dagegen Studien zu informellen Bildungsprozessen. Der Großteil vorliegender (quasi-)experimenteller Studien zeigte einen positiven Effekt auf motivationale Kriterien, während sich Effekte auf die Kompetenzentwicklung häufig nicht statistisch absichern ließen. Eine Gamifizierung der Aktivitäten scheint sich positiv auf Motivation und Interesse auszuwirken, insbesondere wenn die Gamifizierung sich nicht auf die Möglichkeit zum Sammeln von Punkten und Abzeichen beschränkt, sondern künstlerische Prozesse wie die Gestaltung von Avataren oder virtuellen Stempeln einbezieht. Ein zweiter Schwerpunkt für genreübergreifende Arbeiten bezog sich auf die Verwendung digitaler Werkzeuge zum Lernen über fremde Kulturen.

Implikationen: Angesichts der bislang diskutierten Befunde lässt sich folgendes Fazit auf der Ebene formaler Merkmale ziehen: (1) Es liegen viele genreübergreifende Arbeiten vor sowie eine größere Zahl zu den bildenden Künsten, dagegen nur wenige zu anderen klassischen Genres. (2) In Bezug auf die Digitalisierungsgenerationen findet sich wenig zu aktuellen Entwicklungen. (3) Arbeiten zu informellen 
Bildungsprozessen sind selten. (4) Sowohl qualitative als auch die vergleichsweise seltenen quantitativen Arbeiten bleiben in Design wie Analysen oft hinter dem Machbaren zurück. Insbesondere ist bedauerlich, dass sich viele der vorliegenden Studien auf simple Vergleiche digital/analog bei ansonsten identischen Bildungsangeboten bzw. Bildungsprozessen beschränken. Damit wiederholen sie das, was bereits Salomon (1978) für die Medienforschung als „full acceleration in neutral gear" (S. 37) bezeichnet hat.

Angesichts dieser Desiderate verliert die im Ergebnisteil dargestellte, auf inhaltlicher Ebene synthetisierte Evidenz einiges an Durchschlagskraft. Für Effekte auf den Kompetenzerwerb wurde häufig nicht einmal der Versuch einer statistischen Absicherung unternommen. Was bleibt, ist im Wesentlichen ein über die Breite der Interventionen hinweg robuster Effekt auf Motivation und Interesse, von dem jedoch angesichts der gewählten Vergleichsgruppen nicht zweifelsfrei ausgeschlossen werden kann, dass es sich um einen Novitäts-Effekt handelt. Um - im Bild von Salomon (1978) gesprochen - in den nächsten Gang zu schalten, sollte künftige quantitativempirische Forschung zur Digitalisierung in der kulturellen Bildung daher verstärkt Vor- und Nachteile unterschiedlicher Gestaltungsoptionen innerhalb von digitalen Bildungsangeboten in den Blick nehmen. Weitere qualitative Studien können in diesem Zusammenhang einen besonderen Fokus auf die individuellen Erfahrungen der Teilnehmenden legen und daraus Gestaltungshinweise für die Optimierung der verwendeten digitalen Werkzeuge und Aktivitäten ableiten.

\subsection{Limitationen}

Man könnte einwenden, dass es eine große Zahl von Arbeiten mit Relevanz für die digitale kulturelle Bildung gibt, die in der vorliegenden Arbeit nicht berücksichtigt wurde, weil darin nicht explizit von kultureller Bildung die Rede ist (Christ et al. 2019). Allerdings war es gerade Ziel der Arbeit, zu eruieren, inwiefern digitale kulturelle Bildung bereits als eigenständiges Forschungsfeld international sichtbar ist. Deshalb haben wir bewusst lediglich nach denjenigen Arbeiten gesucht, deren Datenbankeintrag über einschlägige Begriffe eine explizite Selbstverortung der Autoren im Feld der Forschung zur kulturellen Bildung erkennen ließ. Eine weitere mögliche Kritik an der vorliegenden Arbeit besteht darin, dass wir unsere Suche auf Scopus und das Fachportal Pädagogik als einschlägige Datenbanken fokussiert haben. Hierzu ist zunächst anzumerken, dass die Suche damit breiter angelegt war als in vielen Reviews aus verschiedensten Themengebieten, die ausschließlich Artikel in Fachzeitschriften berücksichtigen (Crompton et al. 2019; Hopfenbeck et al. 2017; Reisoğlu et al. 2017). Zum anderen ging es uns gerade darum, herauszufinden inwieweit digitale kulturelle Bildung in einschlägigen Literaturdatenbanken sichtbar wird. Schließlich bleibt anzumerken, dass bei der Zusammenstellung der Suchbegriffe neben Englisch lediglich Deutsch Berücksichtigung fand. Dadurch wird der Beitrag von Ländern mit anderen Sprachen unterschätzt. Wenn allerdings bei den deutschen Arbeiten dennoch Lücken deutlich werden, beispielsweise im Bereich der DBR-Forschung, erscheint es umso dringender geboten, auf Abhilfe zu sinnen.

Grundlage für die vorliegende Arbeit war eine Reihe von Begriffsdefinitionen und darauf basierender Kategorisierungen. In diesem Zusammenhang ließe sich dis- 
kutieren, ob eine andere Binnendifferenzierung der Künste als die verwendete nach klassischen Genres sinnvoll gewesen wäre. Angesichts der noch immer starken Orientierung des Feldes der kulturellen Bildung an den klassischen Künsten erschien uns die traditionelle Genreunterteilung jedoch nach wie vor sinnvoll, auch wenn sie bei Arbeiten mit digitalem Bezug hin und wieder an ihre Grenzen stößt. Zugleich ließe sich diskutieren, ob für die Erforschung von Implikationen der digitalen Transformation der Gesellschaft für das Feld der kulturellen Bildung nicht überhaupt eine breitere Definition von Kultur als die anhand der Künste angemessen wäre. Beispielsweise könnten für Kultur und Bildung ebenfalls vermehrt relevante Phänomene wie Digital Making (Smolarczyk und Kröner 2020) oder digital gestütztes Engagement für nachhaltige Entwicklung einbezogen werden. Auch davon haben wir vor dem Hintergrund der aktuellen Ausrichtung der Community der kulturellen Bildungsforschung abgesehen.

Als konfigurierende Forschungssynthese hatte die vorliegende Arbeit die Aufgabe, vorhandene empirische Forschung zur Digitalisierung in der kulturellen Bildung kategorisierend zu kartieren. Die resultierenden Kategorisierungen nach bibliographischen Merkmalen und methodischen Bezügen sowie inhaltlich nach Lebensphase, Formalisierungsgrad, Genres, Digitalisierungsgeneration und zentralen Fragestellungen mögen dabei noch recht grob erscheinen, auch vor dem Hintergrund der im Ergebnisteil erwähnten, teilweise recht heterogenen prototypischen Forschungsfragen innerhalb der einzelnen Facetten. Hier wäre es denkbar gewesen, feiner zu differenzieren, beispielsweise zu den Lebensphasen im Hinblick auf frühe Kindheit oder Emerging Adulthood oder allgemein zur disziplinären und damit einhergehend theoretischen Verortung der Arbeiten. Dies hätte jedoch zu einem Missverhältnis aus Facettenzahl und Menge der zu kategorisierenden Arbeiten geführt. Angesichts der Breite des Feldes mit seiner Vielzahl von Genres, Studiendesigns, Lebensphasen der Probanden, Formalisierungsgrade und digitalen Phänomenen wird deutlich, dass digitale kulturelle Bildung als Feld von weiterer empirischer Forschung nur profitieren kann. Hierbei kann auch aus weiteren theoretischen Analysen zeitversetzt eine Stärkung der empirischen Forschung resultieren (Rosenshine und Furst 1973). Es bleibt zu hoffen, dass das Feld so bald den Reifegrad erreicht, der Metaanalysen zu inhaltlich homogenen Phänomenen der Digitalisierung in der kulturellen Bildung ermöglicht.

\subsection{Fazit}

Sollte angesichts der geringen Gesamtzahl identifizierter Arbeiten konstatiert werden, dass zu wenig empirische Forschung zu digitaler kultureller Bildung stattfindet, insbesondere jenseits der oben genannten Unterrepräsentierung einiger Facetten? Ein derartiges Urteil ist naheliegend, erscheint jedoch angesichts des zuletzt beträchtlichen Zuwachses an Publikationen voreilig. Möglicherweise befinden sich viele interessante Arbeiten derzeit gerade in Vorbereitung, insbesondere in den aktuell laufenden oder erst kürzlich abgeschlossenen einschlägigen BMBF-Förderschwerpunkten. Zudem werden bedingt durch eine entsprechende Fachkultur Forschungsergebnisse nach wie vor oft wenig sichtbar in Form grauer Literatur dokumentiert und gelangen so nicht in die für Scoping Reviews wie die vorliegende Arbeit ein- 
schlägigen nationalen und internationalen Datenbanken. Hier wäre ein Wandel der Publikationskultur hilfreich.

Nach mittlerweile mehr als einer Dekade Forschung zur Digitalisierung in der kulturellen Bildung erscheint es uns an der Zeit, in der ,descriptive-correlationalexperimental-loop“ im Sinne von Rosenshine und Furst (1973, S. 122f.) auch für die Forschung zur digitalen kulturellen Bildung weiter voranzukommen. An den derzeitigen Stand des Diskurses zur Digitalisierung in der kulturellen Bildung sollte sich folglich sowohl weitere qualitativ-empirische Forschung als auch weitere quantitativempirische Arbeit anschließen. In beiden Fällen ist ein Fokus auf starke Designs und systematische Analysen anzustreben. Für die quantitativen Arbeiten sind starke Interventionsdesigns mit detaillierter inferenzstatistischer Analyse ratsam. Qualitative Arbeiten könnten - gerade bei Forschung zur Gestaltung von Bildungsangeboten verstärkt in Studien nach dem DBR-Ansatz eingebettet werden, um die Verzögerung von innovativen Ideen bis zur Anwendungsreife zu verkürzen. Denn was auch immer künftig mit Schlagworten wie Web X.0 (Benito-Osorio et al. 2013) versehen werden mag - die Digitalisierung als allgemeiner Trend wird nicht darauf warten, bis die experimentelle Forschung zu den jeweils vorangegangenen Web-Generationen abgeschlossen ist. Vor allem aber sollte empirische Forschung zu digitaler kultureller Bildung jenseits naiver digital/analog-Vergleiche verstärkt auf das Herausarbeiten von Gestaltungsprinzipien und Gelingensbedingungen erfolgreicher formaler und nonformaler Angebote bzw. informeller Prozesse zielen.

In jedem Fall spricht vieles dafür, zukünftig auch und gerade in Deutschland sowohl die empirische Forschung zu denjenigen Facetten der Forschung zur Digitalisierung in der kulturellen Bildung zu stärken, die bislang eher sporadisch in den Blick genommen wurden, als auch die Ergebnisse verstärkt in relevanten internationalen Fachzeitschriften zu publizieren. Die sich so ergebende höhere internationale Sichtbarkeit würde der an sich recht breit aufgestellten deutschen Community der Forschung zur Kulturellen Bildung zu größerer Reichweite verhelfen. Wir hoffen, dass die vorliegende Forschungssynthese dazu beiträgt, entsprechende Anschlussstellen zu identifizieren.

Danksagung Wir danken André Fleischmann, Maximilian Malter, Ruth Martini und Gerlinde Steinbinder für die tatkräftige Unterstützung bei der Sichtung der Arbeiten und der Kategorisierung der eingeschlossenen Publikationen.

Förderung Das dieser Arbeit zugrundeliegende Projekt (Digitalisierung in der kulturellen Bildung - Ein Metaprojekt, DiKuBi-Meta, Projektleitung Benjamin Jörissen und Stephan Kröner), wurde gefördert durch das Bundesministerium für Bildung und Forschung, Förderkennzeichen 01JKD1711.

Funding Open Access funding enabled and organized by Projekt DEAL.

Open Access Dieser Artikel wird unter der Creative Commons Namensnennung 4.0 International Lizenz veröffentlicht, welche die Nutzung, Vervielfältigung, Bearbeitung, Verbreitung und Wiedergabe in jeglichem Medium und Format erlaubt, sofern Sie den/die ursprünglichen Autor(en) und die Quelle ordnungsgemäß nennen, einen Link zur Creative Commons Lizenz beifügen und angeben, ob Änderungen vorgenommen wurden.

Die in diesem Artikel enthaltenen Bilder und sonstiges Drittmaterial unterliegen ebenfalls der genannten Creative Commons Lizenz, sofern sich aus der Abbildungslegende nichts anderes ergibt. Sofern das betreffende Material nicht unter der genannten Creative Commons Lizenz steht und die betreffende Handlung 
nicht nach gesetzlichen Vorschriften erlaubt ist, ist für die oben aufgeführten Weiterverwendungen des Materials die Einwilligung des jeweiligen Rechteinhabers einzuholen.

Weitere Details zur Lizenz entnehmen Sie bitte der Lizenzinformation auf http://creativecommons.org/ licenses/by/4.0/deed.de.

\section{Literatur}

Abend, P., \& Beil, B. (2017). Spielen in mediatisierten Welten - Editor-Games und der Wandel der zeitgenössischen Digitalkulturen. In F. Krotz, C. Despotović \& M. M. Kruse (Hrsg.), Mediatisierung als Metaprozess (S. 303-321). Wiesbaden: Springer VS.

Bamford, A. (2010). Der Wow-Faktor. Eine weltweite Analyse der Qualität künstlerischer Bildung. Münster: Waxmann.

Batteux, C. (1976). Einschränkung der schönen Künste auf einen einzigen Grundsatz. Hildesheim: Olms. https://reader.digitale-sammlungen.de/de/fs1/object/display/bsb10812914_00005.html. (Originalarbeit erschienen 1747).

Benito-Osorio, D., Peris-Ortiz, M., Armengot, C. R., \& Colino, A. (2013). Web 5.0: the future of emotional competences in higher education. Global Business Perspectives, 1, 274-287. https://doi.org/10.1007/ s40196-013-0016-5.

BMBF (2017). Bekanntmachung zur Richtlinie zur Förderung von Forschung zu „Digitalisierung im Bildungsbereich - Grundsatzfragen und Gelingensbedingungen“. Bundesanzeiger vom 26.09.2017. https://www.bmbf.de/foerderungen/bekanntmachung-1420.html.

BMBF (2019). Bekanntmachung zur Richtlinie zur Förderung von Forschungsvorhaben zur kulturellen Bildung in ländlichen Räumen. Bundesanzeiger vom 08.01.2019. https://www.bmbf.de/foerderungen/ bekanntmachung-2232.html.

Breiter, A., Welling, S., \& Schulz, A.H. (2012). Mediatisierung schulischer Organisationskulturen. In F. Krotz \& A. Hepp (Hrsg.), Mediatisierte Welten (S. 113-135). Wiesbaden: Springer VS. https:// doi.org/10.1007/978-3-531-94332-9_5.

Brickenkamp, R. (1990). Die Generelle Interessen-Skala (GIS). Göttingen: Hogrefe.

Bromme, R., Prenzel, M., \& Jäger, M. (2014). Empirische Bildungsforschung und evidenzbasierte Bildungspolitik. Zeitschrift für Erziehungswissenschaft, 17(1), 3-54. https://doi.org/10.1007/s11618014-0514-5.

Choudhury, N. (2014). World wide web and its journey from web 1.0 to web 4.0. International Journal of Computer Science and Information Technologies, 5, 8096-8100. http://ijcsit.com/docs/Volume\%205/ vol5issue06/ijcsit20140506265.pdf.

Christ, A., Penthin, M., \& Kröner, S. (2019). Big data and digital aesthetic, arts, and cultural education: hot spots of current quantitative research. Social Science Computer Review. https://doi.org/10.1177/ 0894439319888455.

Costa, P.T., \& McCrae, R.R. (1995). Domains and facets: hierarchical personality assessment using the revised NEO personality inventory. Journal of personality assessment, 64, 21-50. https://doi.org/10. 1207/s15327752jpa6401_2.

Couldry, N. (2014). Mediatization and the future of field theory. In K. Lundby (Hrsg.), Mediatization of communication (S. 227-245). Berlin: De Gruyter.

Creswell, J.W. (2014). Research design. Qualitative, quantitative, and mixed methods approaches (4. Aufl.). Los Angeles: SAGE.

Crompton, H., Burke, D., \& Lin, Y.-C. (2019). Mobile learning and student cognition: a systematic review of PK-12 research using bloom's taxonomy. British Journal of Educational Technology, 50, 684-701. https://doi.org/10.1111/bjet.12674.

Dicks, L. V., Walsh, J. C., \& Sutherland, W. J. (2014). Organising evidence for environmental management decisions: a '4S' hierarchy. Trends in ecology \& evolution, 29, 607-613.

Fink, T., Hill, B., Reinwand, V.-I., \& Wenzlik, A. (2012). Begrifflich, empirisch, künstlerisch: Forschung im Feld der Kulturellen Bildung. In T. Fink (Hrsg.), Die Kunst, über Kulturelle Bildung zu forschen (Kulturelle Bildung, Bd. 29, S. 9-21). München: kopaed.

Frese, M., \& Brodbeck, F.C. (1989). Computer in Büro und Verwaltung: psychologisches Wissen für die Praxis. Berlin: Springer.

Gough, D., Oliver, S., \& Thomas, J. (2017). An introduction to systematic reviews (2. Aufl.). Los Angeles: SAGE. 
Grenz, T., \& Pfadenhauer, M. (2017). Kulturen im Wandel: Zur nonlinearen Brüchigkeit von Mediatisierungsprozessen. In F. Krotz, C. Despotović \& M. M. Kruse (Hrsg.), Mediatisierung als Metaprozess (S. 187-210). Wiesbaden: Springer VS.

Hamari, J., \& Keronen, L. (2017). Why do people play games? A meta-analysis. International Journal of Information Management, 37, 125-141. https://doi.org/10.1016/j.ijinfomgt.2017.01.006.

Hasselhorn, M., \& Krampen, G. (2019). Monitoring des Beitrags der Psychologie zur interdisziplinären Bildungsforschung: Szientometrische Analysen zur Forschung aus dem deutschsprachigen im Vergleich zum angloamerikanischen Bereich. Schweizerische Zeitschrift für Bildungswissenschaften, 41, 13-35. https://doi.org/10.24452/SJER.41.1.4.

Hepp, A., \& Lehmann-Wermser, A. (2013). Transformation des Kulturellen. Zur Einleitung in den Band. In A. Hepp \& A. Lehmann-Wermser (Hrsg.), Transformation des Kulturellen (S. 9-18). Wiesbaden: Springer VS. https://doi.org/10.1007/978-3-531-19239-0_11.

Hopfenbeck, T. N., Lenkeit, J., El Masri, Y., Cantrell, K., Ryan, J., \& Baird, J.-A. (2017). Lessons learned from PISA. A systematic review of peer-reviewed articles on the Programme for International Student Assessment. Scandinavian Journal of Educational Research, 10, 1-21. https://doi.org/10.1080/ 00313831.2016 .1258726$.

Hugger, K.U. (2013). Bildung im gegenwärtigen Mediatisierungsprozess. Kulturelle Bildung Online. https://doi.org/10.25529/92552.451.

v. Humboldt, W. (1904). Aesthetische Versuche. Erster Teil (Gesammelte Schriften: Ausgabe der Preussischen Akademie der Wissenschaften). Berlin: B. Behr's. https://archive.org/search.php? query=external-identifier\%3A\%22urn\%3Aoclc\%3Arecord\%3A1045571851\%22. (Originalarbeit erschienen 1799).

Hurrelmann, K. (2003). Der entstrukturierte Lebenslauf. Die Auswirkungen der Expansion der Jugendphase. ZSE: Zeitschrift für Soziologie der Erziehung und Sozialisation, 23, 115-126. https://nbnresolving.org/urn:nbn:de:0111-opus-57522.

Hussy, W., Schreier, M., \& Echterhoff, G. (2013). Forschungsmethoden in Psychologie und Sozialwissenschaften für Bachelor (2. Aufl.). Berlin: Springer.

Ijdens, T. (2016). Monitoring National Arts Education Systems (MONAES): some results of two surveys among arts education experts around the world. Utrecht: Netherlands Centre of Expertise for Cultural Education and Amateur Arts. https://lkca.nl/wp-content/uploads/2020/01/2016_monaes_ exploratory_analysis.pdf. Zugegriffen: 29. Januar 2021.

Iske, S., \& Meder, N. (2010). Lernprozesse als Performanz von Bildung in den Neuen Medien. In K. U. Hugger \& M. Walber (Hrsg.), Digitale Lernwelten (S. 21-37). Wiesbaden: Springer VS.

Jeong, H., Hmelo-Silver, C. E., \& Yu, Y. (2014). An examination of CSCL methodological practices and the influence of theoretical frameworks 2005-2009. International Journal of Computer-Supported Collaborative Learning, 9, 305-334. https://doi.org/10.1007/s11412-014-9198-3.

Jörissen, B. (2018). Subjektivation und ästhetische Bildung in der post-digitalen Kultur. Vierteljahrsschrift für wissenschaftliche Pädagogik, 94, 51-70. https://doi.org/10.30965/25890581-09401006.

Jörissen, B., \& Unterberg, L. (2019). Digitalität und Kulturelle Bildung. In B. Jörissen, S. Kröner \& L. Unterberg (Hrsg.), Forschung zur Digitalisierung in der Kulturellen Bildung (S. 11-24). München: kopaed.

Jörissen, B., Kröner, S., \& Unterberg, L. (Hrsg.). (2019). Forschung zur Digitalisierung in der Kulturellen Bildung. München: kopaed.

Keuchel, S. (2012). Empirische Forschung in der Kulturellen Bildung unter besonderer Berücksichtigung quantitativer Forschungsansätze. In T. Fink (Hrsg.), Die Kunst, über Kulturelle Bildung zu forschen (Kulturelle Bildung, Bd. 29, S. 36-50). München: kopaed.

Krieck, E. (1930). Erziehungsphilosophie (Teil 2). München: Oldenbourg.

Kristeller, P. O. (1951). The modern system of the arts. A study in the history of aesthetics part I. Journal of the History of Ideas, 12, 496-527. https://www.jstor.org/stable/2707484?seq=1.

Kröner, S. (2013). Kulturelle Partizipation bei Jugendlichen als Feld der Person-Umwelt-Transaktion. In A. Scheunpflug \& M. Prenzel (Hrsg.), Kulturelle und ästhetische Bildung (Zeitschrift für Erziehungswissenschaft: Sonderheft 21, S. 233-256). https://doi.org/10.1007/s11618-013-0432-y.

Kröner, S., \& Dickhäuser, O. (2009). Die Rolle von Eltern, Peers und intrinsischem Wert für die rezeptive hochkulturelle Praxis von Gymnasiasten der Sekundarstufe II. Zeitschrift für Pädagogische Psychologie, 23, 53-63. https://doi.org/10.1024/1010-0652.23.1.53.

Kröner, S., Christ, A., \& Penthin, M. (2019). Forschungssynthesen zur Digitalisierung in der kulturellen Bildung. In B. Jörissen, S. Kröner \& L. Unterberg (Hrsg.), Forschung zur Digitalisierung in der Kulturellen Bildung (S. 215-228). München: kopaed. 
Kröner, S., Christ, A., \& Penthin, M. (2021). Stichwort: Digitalisierung in der kulturell-ästhetischen Bildung - eine konfigurierende Forschungssynthese (Stichwort_Kategoriesierung.csv) [Datensatz und Kategorisierungen]. https://doi.org/10.17605/OSF.IO/KX89G.

Kröner, S., Lüdtke, O., Maaz, K., Trautwein, U., \& Köller, O. (2008). Wer geht ins Theater? Künstlerisches Interesse und Offenheit für Erfahrung als Prädiktoren für Veränderungen kultureller Partizipation in der Emerging Adulthood. Zeitschrift für Entwicklungspsychologie und Pädagogische Psychologie, 40, 100-110. https://doi.org/10.1026/0049-8637.40.2.100.

Krotz, F. (2017). Mediatisierung: Ein Forschungskonzept. In F. Krotz, C. Despotovic \& M. M. Kruse (Hrsg.), Mediatisierung der Gesellschaft. Medien - Kultur - Kommunikation (S. 13-32). Wiesbaden: Springer VS.

Krotz, F., \& Hepp, A. (Hrsg.). (2012). Mediatisierte Welten. Wiesbaden: Springer VS.

Krotz, F., Despotović, C., \& Kruse, M. M. (Hrsg.). (2017). Mediatisierung als Metaprozess: Transformationen, Formen der Entwicklung und die Generierung von Neuem. Wiesbaden: Springer VS.

Krupp-Schleußner, V., \& Lehmann-Wermser, A. (2016). An instrument for every child. A study on longterm effects of extended music education in German primary schools. Music Education Research, 13, 1-15. https://doi.org/10.1080/14613808.2016.1249361.

Kuypers, H.W., \& Leyendecker, B. (1982). Erwachsenenbildung in der Praxis. Didaktik und Methodik. Bad Heilbrunn: Klinkhardt.

La Belle, T. J. (1982). Formal, nonformal and informal education: a holistic perspective on lifelong learning. International Review of Education, 28, 159-175. https://doi.org/10.1007/BF00598444.

Lavranos, C., Kostagiolas, P., Korfiatis, N., \& Papadatos, J. (2016). Information seeking for musical creativity: a systematic literature review. Journal of the Association for Information Science and Technology, 67, 2105-2117. https://doi.org/10.1002/asi.23534.

Liebau, E., Jörissen, B., Hartmann, S., Lohwasser, D., Werner, F., Klepacki, L., \& Wagner, E. (2013). Forschung zur kulturellen Bildung. In Bundesministerium für Bildung und Forschung (Hrsg.), Perspektiven der Forschung zur kulturellen Bildung (S. 13-18). Bonn: Bundesministerium für Bildung und Forschung.

McAdams, D. P. (2006). The redemptive self. Stories Americans live by. Oxford: Oxford University Press.

Penthin, M., Christ, A., \& Kröner, S. (2018). Quantitative Forschungssynthese zur Digitalisierung in der Kulturellen Bildung (DiKuBi). Poster präsentiert auf dem 51. Kongress der Deutschen Gesellschaft für Psychologie, Frankfurt am Main. https://osf.io/j89bc.

Penthin, M., Fritzsche, E.S., \& Kröner, S. (2017). Bereichsspezifische Determinanten außerschulischer musikalischer Aktivitäten von Grundschulkindern. Beiträge Empirischer Musikpädagogik, 8, 1-30. https://b-em.info/index.php/ojs/article/view/147.

Petticrew, M., \& Roberts, H. (2012). Systematic reviews in the social sciences. A practical guide (12. Aufl.). Malden: Blackwell.

Peura, P. I., Viholainen, H. J. K., Aro, T. I., Räikkönen, E. M., Usher, E. L., Sorvo, R. M. A., Klassen, R. M., \& Aro, M. T. (2019). Specificity of reading self-efficacy among primary school children. The Journal of Experimental Education, 87, 496-516. https://doi.org/10.1080/00220973.2018.1527279.

Projektgruppe „Forschung zur Kulturellen Bildung in Deutschland“ (2014). Was wir sehen und was wir nicht sehen. Zum Stand der Forschung über Kulturelle Bildung in Deutschland. In E. Liebau, B. Jörissen \& L. Klepacki (Hrsg.), Forschung zur Kulturellen Bildung. Grundreflexionen und empirische Befunde (Kulturelle Bildung, Bd. 39, S. 177-214). München: kopaed.

Rammstedt, B. (2019). Der Einfluss kultureller Bildung auf die Entwicklung sozio-emotionaler Fähigkeiten - eine systematische Analyse des Forschungsstands. Tagung des Rates für Kulturelle Bildung, Münster, 14. März 2019.

Rat für kulturelle Bildung (2013). Alles immer gut - Mythen kultureller Bildung. https://www.ratkulturelle-bildung.de/fileadmin/user_upload/pdf/RKB_ALLES_IMMER_GUT_Einzelseiten.pdf. Zugegriffen: 29. Januar 2021.

Reckwitz, A. (2004). Die Kontingenzperspektive der „Kultur“. Kulturbegriffe, Kulturtheorien und das kulturwissenschaftliche Forschungsprogramm. In B. Liebsch, J. Straub, F. Jaeger \& J. Rüsen (Hrsg.), Handbuch der Kulturwissenschaften: Themen und Tendenzen (Bd. 3, S. 1-12). Stuttgart: J.B. Metzler.

Reisoğlu, I., Topu, B., Yılmaz, R., Karakuş, Y. T., \& Göktaş, Y. (2017). 3D virtual learning environments in education: a meta-review. Asia Pacific Education Review, 18, 81-100. https://doi.org/10.1007/ s12564-016-9467-0.

Robinsohn, S. B. (1971). Bildungsreform als Revision des Curriculum und ein Strukturkonzept für Curriculumentwicklung. Neuwied: Luchterhand. 
Rosenshine, B., \& Furst, N. (1973). The use of direct observation on study teaching. In N.L. Gage \& R.M.W. Travers (Hrsg.), Second handbook of research on teaching (S. 122-183). Chicago: Rand McNally.

Sala, G., \& Gobet, F. (2017). When the music's over. Does music skill transfer to children's and young adolescents' cognitive and academic skills? A meta-analysis. Educational Research Review, 20, 55-67. https://doi.org/10.1016/j.edurev.2016.11.005.

Salomon, G. (1978). On the future of media research: no more full acceleration in neutral gear. ECTJ, 26, 37-46. https://doi.org/10.1007/BF02766573.pdf.

Schröder, S. (2019). Transfereffekte zwischen Musik und Sprache: Mythos oder Realität. Tagung des Rates für Kulturelle Bildung, Münster, 14. März 2019.

Schüller, E.M., Birnbaum, L., \& Kröner, S. (2017). What makes elementary school students read in their leisure time? Development of a comprehensive questionnaire. Reading Research Quarterly, 52, 161-175. https://doi.org/10.1002/rrq.164.

Schulze, G. (2000). Die Erlebnis-Gesellschaft. Kultursoziologie der Gegenwart (8. Aufl.). Frankfurt a. M.: Campus.

Smolarczyk, K., \& Kröner, S. (2020). FabLabs, Makerspaces \& Co: Eine Forschungssynthese zum digitalen Making mit Kindern und Jugendlichen. Poster präsentiert auf der 8. Tagung der Gesellschaft für Empirische Bildungsforschung (GEBF), Potsdam.

Stuckert, M., Rohde, J., Züchner, I., \& Thole, W. (2018). Jugendkunstschulen und kulturpädagogische Projekte als Orte der Bildung: Zentrale Befunde eines Forschungsprojektes. Kulturelle Bildung Online. https://doi.org/10.25529/92552.101.

Thiese, M. S. (2014). Observational and interventional study design types; an overview. Biochemia medica, 24, 199-210. https://hrcak.srce.hr/125364.

Timm, S., Costa, J., Kühn, C., \& Scheunpflug, A. (Hrsg.). (2020). Kulturelle Bildung - Theoretische Perspektiven, methodologische Herausforderungen, empirische Befunde. Münster: Waxmann.

Torgerson, C., Hall, J., \& Lewis-Light, K. (2017). Systematic reviews. In R. Coe, M. Waring, L. V. Hedges \& J. Arthur (Hrsg.), Research methods and methodologies in education (2. Aufl., S. 166-179). Los Angeles: SAGE.

Tromp, J., Le, C., Le, B., \& Le, D. N. (2018). Massively multi-user online social virtual reality systems: ethical issues and risks for long-term use. In A. S. Ashour, V. Bhatnagar \& M. S. Bouhlel (Hrsg.), Social networks science: design, implementation, security, and challenges (S. 131-149). Cham: Springer.

Troyer, M., Kim, J. S., Hale, E., Wantchekon, K. A., \& Armstrong, C. (2019). Relations among intrinsic and extrinsic reading motivation, reading amount, and comprehension: a conceptual replication. Reading and Writing, 32, 1197-1218. https://doi.org/10.1007/s11145-018-9907-9.

Tylor, E. B. (2010). Primitive culture: researches into the development of mythology, philosophy, religion, language, art and custom. London: J. Murray. Originalarbeit erschienen 1871.

Valenzuela, R., \& Codina, N. (2014). Habitus and flow in primary school musical practice: relations between family musical cultural capital, optimal experience and music participation. Music Education Research, 16, 505-520. https://doi.org/10.1080/14613808.2013.859660.

VDI (2000). VDI Richtlinie 3780:2000-09. Technikbewertung - Begriffe und Grundlagen. Düsseldorf: Beuth.

Walmsley, B. (2011). Why people go to the theatre: a qualitative study of audience motivation. Journal of Customer Behaviour, 10, 335-351. https://doi.org/10.1362/147539211X13210329822545.

Wehner, J., Passoth, J.H., \& Sutter, T. (2017). Medien, Musik und Algorithmen - Zur Publikumsvermessung im Internet. In F. Krotz, C. Despotović \& M. M. Kruse (Hrsg.), Mediatisierung als Metaprozess (S. 233-256). Wiesbaden: Springer VS. 